Ronald P. Muraro

EDIS FE629

W. C. Oswalt

http://edis.ifas.ufl.edu/FE629

\title{
Budgeting Costs and Returns for Central Florida Citrus Production, 2004-05
}
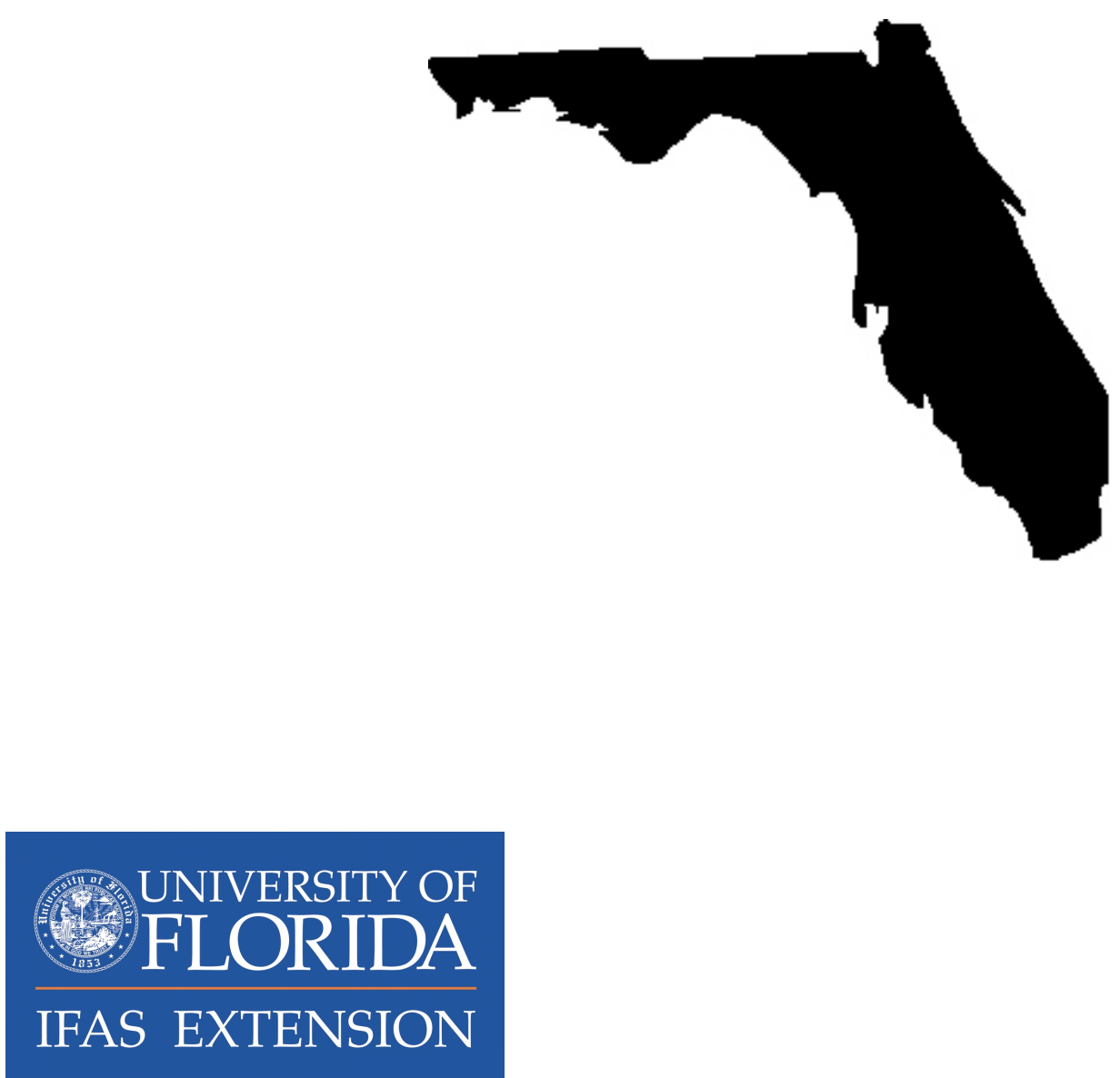

Institute of Food and Agricultural Sciences

Food and Resource Economics Department

Florida Agricultural Experiment Station

Florida Cooperative Extension Service

Gainesville, FL 32611 


\section{ABSTRACT}

Estimated costs and returns of growing round oranges in the Central Florida citrus area are presented for the twenty-second consecutive year. The Central Florida citrus area refers primarily to Polk and Highlands counties. The format presented may be used by individual growers to budget costs and returns, utilizing individual data on specific groves.

Key words: citrus, Central Florida, budgeting, costs and returns.

NOTE: The Central Florida production area refers to Polk and Highlands counties. However, the costs presented in this report are applicable to other counties such as Hardee, Hillsborough, Lake and Orange counties.

The budgeted cost information presented herein is the most current available. The budget cost items have been revised to reflect current grove practices being used by growers-e.g., chemical mowing, different spray materials and rates of fertilization, microsprinkler irrigation, more reset trees, etc. The 2004-2005 budgets reflect major cost increases in all production inputs: fuel averaged a $22 \%$ increase; fertilizer products increased $15 \%$; chemicals averaged an $8 \%$ increase; and equipment operation costs increased $7 \%$. Along with the increased costs, three major hurricanes (storms) during August and September 2004 resulted in wide tree damage and fruit loss. The Indian River region experienced fruit loss of $70 \%$ to $80 \%$ on red and white grapefruit, respectively. Hamlin orange losses in the Central Florida (ridge) region were $30 \%$ to $40 \%$ with Valencia orange losses between $20 \%$ and $30 \%$. The only citrus growing region that was not significantly affected by the three storms was the Southwest Florida citrus region. As a result of the excessive fruit loss, the per box, per pound solid and per carton costs for the Indian River and Central (ridge) growing regions were substantially higher than in recent years.

The budget costs in this report represent a custom-managed operation. Therefore, all equipment costs are based on the average custom rate costs, and a 10 percent handling and supervision charge is added to the material cost.

Although the estimated annual per acre grove costs listed are representative for a mature citrus grove $(10+$ years old), the grove care costs for a specific grove site may differ depending upon the tree age, tree density and the grove practices performed; e.g., spot herbicide for grass/brush regrowth under trees could add an additional $\$ 15.34$ per acre; Diaprepes control could add $\$ 84.18$ per acre for each foliar application; extensive tree loss due to blight or tristeza could substantially increase the tree replacement and care costs; spray applications to control citrus leafminer and nematicide applications such as Temik (\$131.11/acre) could increase the total cultural costs per acre above the average costs shown in the comparative budgets; travel and set-up costs may vary due to size of the citrus grove and distance from the grove equipment barn and could add $\$ 28.86$ per acre; etc.

\section{ACKNOWLEDGEMENTS}

Appreciation is extended to Mrs. Jane Wilson for typing the final draft of this manuscript.

Appreciation is also extended to the citrus growers and production managers of the Central

Florida citrus production area who provided suggestions for the revision of this manuscript. 


\section{TABLE OF CONTENTS}

$\underline{\text { Page }}$

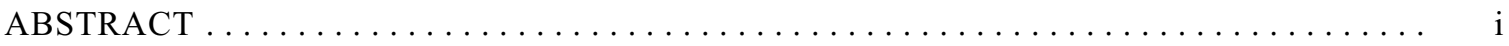

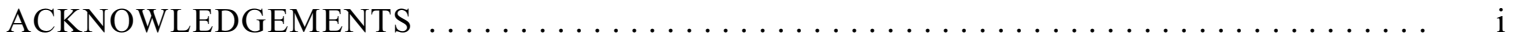

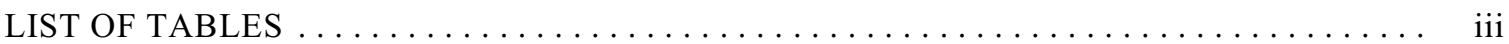

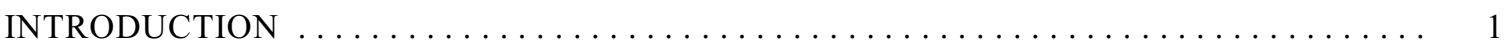

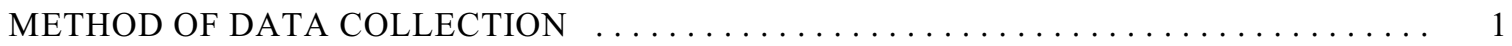

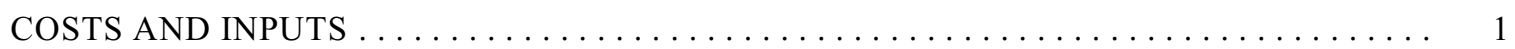

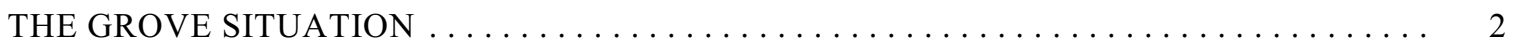

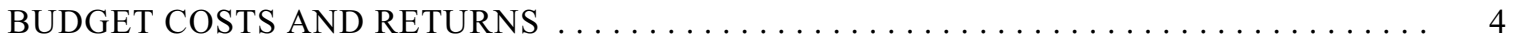

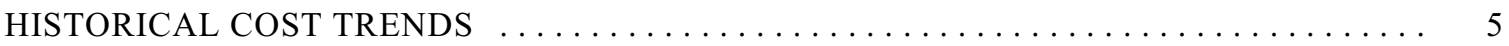

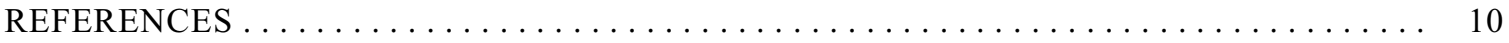

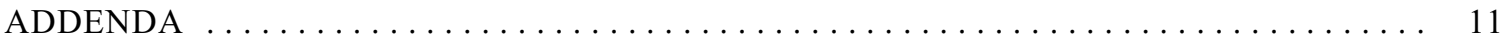

NOTE: The ADDENDA include a Listing of Grove Care Programs for Central Florida Citrus Production for Both Round Oranges and Grapefruit; 2005 custom rate summary report; cost of establishing a citrus grove; etc. Page 11 is a list of the tables included in the ADDENDA. 


\section{LIST OF TABLES}

$\underline{\text { Table }}$

$\underline{\text { Page }}$

1 Schedule of production practices and budget items for a Central Florida citrus grove, 2004-05

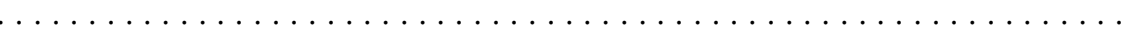

2 Calculation of normal production per acre, $2004-05 \ldots \ldots \ldots \ldots \ldots \ldots \ldots \ldots$

3 Estimated annual per acre costs and returns for a mature, Valencia orange grove producing for the processed market, Central Florida area, $2004-05$. . . . . . . . . . .

4 Estimated total delivered-in cost for Central Florida (Ridge) Valencia oranges grown for the processed juice market under three cultural cost programs, 2004-05 . . . . . . . .

5 Estimated annual per acre costs and returns and 5-year average costs and returns for a mature, Valencia orange grove producing citrus for processing in the Central Florida

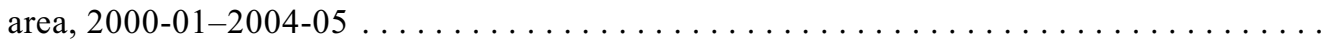

6 Estimated annual per acre costs and returns and 5-year average costs and returns (adjusted to 2005 dollars) for a mature, Valencia orange grove producing citrus for processing in the Central Florida area, 2000-01-2004-05 . . . . . . . . . . . . . . 


\title{
BUDGETING COSTS AND RETURNS FOR CENTRAL FLORIDA CITRUS PRODUCTION, 2004-05
}

\author{
Ronald P. Muraro and W. C. Oswalt
}

\section{INTRODUCTION}

Budget analysis provides the basis for many grower decisions. Budget analysis can be used to calculate potential profits from an operation, to determine cash requirements for an operation, and to determine break-even prices. This report presents a budget constructed from current data and serves as a format for growers to analyze costs and returns from their individual records. During the 1980's, several freezes occurred which changed the character of the Central Florida citrus production area. The December 1983 and January 1985 freezes caused extensive tree and acreage losses in north central counties such as Lake and Orange counties. The December 1989 freeze resulted in severe tree damage and tree loss in North and Central Polk County. Thus, Central Florida in this report refers primarily to Polk and Highlands counties.

The 2004-2005 budgets reflect major cost increases in all production inputs: fuel averaged a $22 \%$ increase; fertilizer products increased 15\%; chemicals averaged an $8 \%$ increase; and equipment operation costs increased 7\%. Along with the increased costs, three major hurricanes (storms) during August and September 2004 resulted in wide tree damage and fruit loss. The Indian River region experienced fruit loss of $70 \%$ to $80 \%$ on red and white grapefruit, respectively. Hamlin orange losses in the Central Florida (ridge) region were $30 \%$ to $40 \%$ with Valencia orange losses between $20 \%$ and $30 \%$. The only citrus growing region that was not significantly affected by the three storms was the Southwest Florida citrus region. As a result of the excessive fruit loss, the per box, per pound solid and per carton costs for the Indian River and Central (ridge) growing regions were substantially higher than in recent years.

\section{METHOD OF DATA COLLECTION}

The data presented here were developed by surveying custom operators, input suppliers, growers, colleagues at the Citrus Research and Education Center in Lake Alfred, and County Extension Citrus Agents in the Central Florida production region. The survey is conducted annually in February and March.

RONALD P. MURARO is a Professor of Food and Resource Economics and Extension Farm Management Economist stationed at the Citrus Research and Education Center, Lake Alfred. W. C. OSWALT is a Multi-county Extension Agent, Citrus in Polk and Hillsborough Counties, Bartow. 


\section{COSTS AND INPUTS}

Costs for various production inputs are those collected from citrus growers as well as the average of the data obtained from annual custom rate, chemical, and fertilizer surveys. Growers' costs are shown in the ADDENDA, Tables 1-A through 7-A. The custom rate costs are shown in Table 8-A and the various chemical and fertilizer costs are shown in Table 9-A and 10-A in the ADDENDA. The budget costs represent a custom-managed operation. Therefore, all equipment costs are based upon the average custom-rate costs and a 10 percent handling and supervision charge is added to the material cost.

Although brand names are used in many of the tables in the ADDENDA, this does not imply endorsement by the University of Florida. It is merely an attempt to depict typical production practices.

All tables have a column reserved for the individual growers to insert data from a particular grove allowing a comparison of the grower's costs with those presented.

\section{THE GROVE SITUATION}

Production practices for a Central Florida round orange grove are shown in Table 1 with times during the year when they would likely be performed. There are two benefits to developing such a table for an individual grove. First, it shows what work is needed and when, so that operations can be planned well in advance. Second, it can be helpful if an annual cash flow analysis is developed to plan financing. The individual grower may benefit from developing a plan for a particular grove.

Specific production practices vary from grove to grove making it difficult to define a "typical" grove. Many combinations of practices and various tree variety combinations produce acceptable yields and returns. Although the example represents a Valencia orange grove, the cost and return data are designed to be applicable to most grove situations. A grower, realtor, or land appraiser can substitute individual grove costs and expected returns into the budget format and develop a budget for a particular grove. A "your cost" column is appropriately provided for this purpose in subsequent tables.

In the following budget, above average management and cultural practices are assumed. Beyond this general assumption, the following specifics are assumed.

1. A $10+$ year-old, low volume-irrigated grove;

2. Variety is Valencia round orange;

3. Tree loss is 3 percent annually;

4. Trees are pulled and replaced when production falls below 50 percent of expected yield;

5. Production is for processed use;

6. Tree density is 112 trees per acre; and

7. Custom-caretaker is providing grove management. 


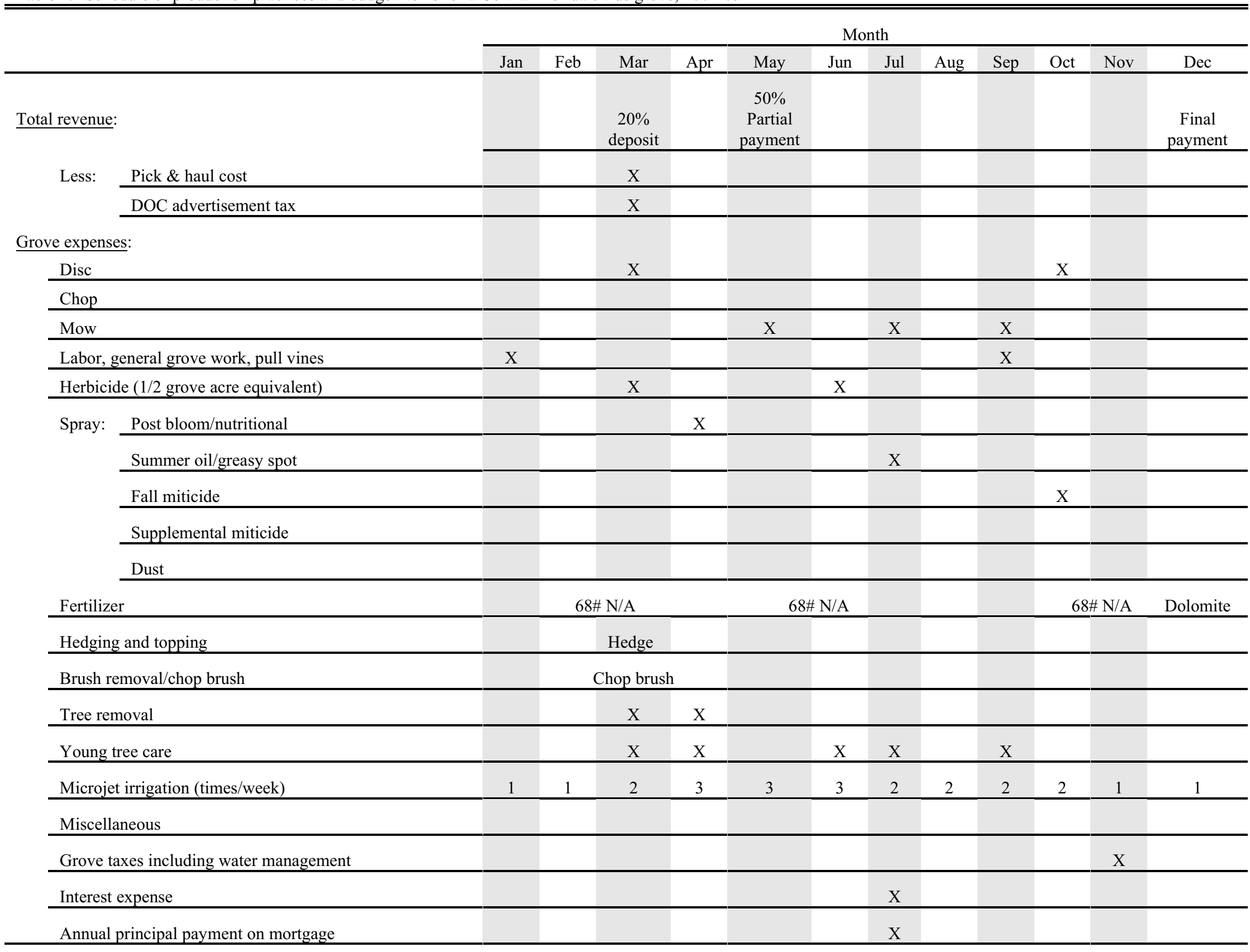

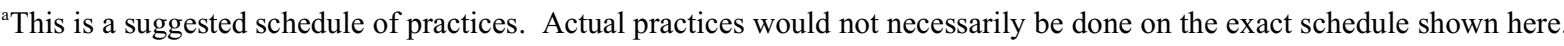


As a result of tree losses and replacement, the tree ages will vary. The budget reflects the following age distribution:

\begin{tabular}{rlc} 
& \multicolumn{1}{c}{ Situation } & $\begin{array}{c}\text { Yield } \\
\text { Boxes } / \text { tree }\end{array}$ \\
\cline { 2 - 2 } $3 \%$ & pulled and reset & 0.0 \\
$3 \%$ & 1 year old & 0.0 \\
$3 \%$ & 2 years old & 0.0 \\
$3 \%$ & 3 years old & 0.7 \\
$3 \%$ & 4 years old & 0.9 \\
$45 \%$ & 5 -19 years old & 4.0 \\
$3 \%$ & producing 50\% of expected yield & 2.9 \\
$37 \%$ & mature producing & 5.5
\end{tabular}

Calculation of normal production per acre is shown in Table 2. Note that the proportion-of-treesby-age column only adds to 91 percent since 9 percent of the trees are non-bearing. The impact of the three hurricanes in 2004 is reflected in a $25 \%$ reduction in normal yields.

Table 2.--Calculation of normal production per acre, 2004-05

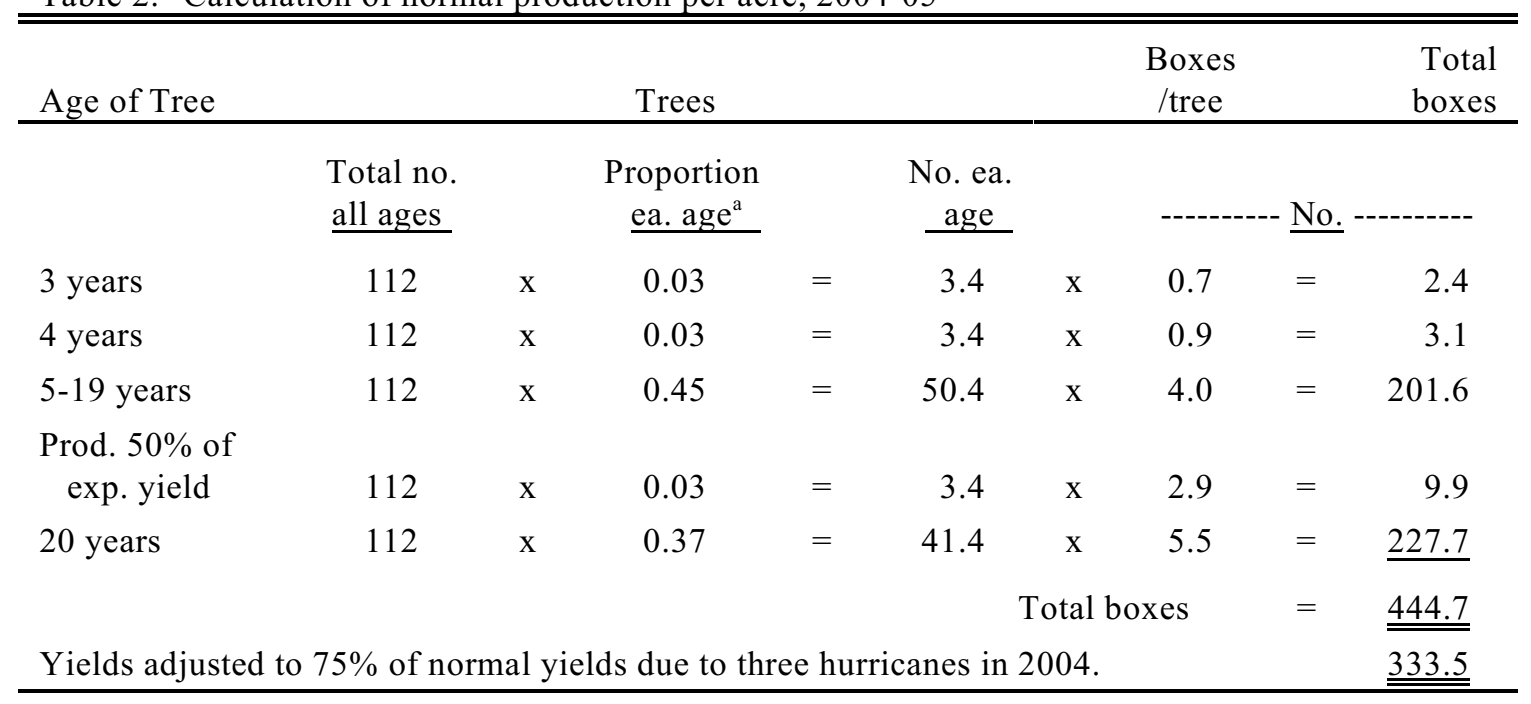

${ }^{\text {a}}$ Proportion adds up to 0.91 (91 percent) as 9 percent of the trees were non-bearing (pulled and reset, 1 and 2 year old trees).

\section{BUDGET COSTS AND RETURNS}

The estimated budget costs and returns for the Central Florida grove situation are shown in Table 3. The budgeted costs represent one possible citrus production program and were selected from the costs shown in the ADDENDA tables. The gross revenue estimates are based on the projected yields in Table 3 and estimated preliminary on-tree prices for the 2004-05 season. Reset costs, alternative cost scenarios, harvesting and packing charges can be found in Tables 11-A through 14-A in the ADDENDA. 
Also, historical on-tree prices for selected Florida citrus varieties are shown in Table 15-A of the ADDENDA.

As shown in Table 3, the total revenue for processed-market Valencia oranges is estimated to be $\$ 1,425.24$ per acre. Total specified costs are $\$ 895.43$ and are comprised of grove care costs of $\$ 847.43$, plus management cost of $\$ 48.00$. Return to land and trees of $\$ 529.81$ represents net return above variable costs. At 300 and 500 boxes per acre, respectively, the break-even price required to cover grove care costs for Valencia oranges range from $\$ 2.83$ to $\$ 1.70$ per box on-tree and $\$ 0.81$ to $\$ 0.64$ per pounds solids delivered-in.

Ad valorem taxes, and overhead and administrative costs (such as water drainage district taxes, crop insurance, and other grower assessments) can add up to 12 percent to the total grove care costs. These costs vary from grove to grove depending on age, location, variety of fruit, etc. and should be considered in arriving at net return to land, trees and ownership (total return minus total costs). Harvest costs (pick, roadside, and hauling costs) also add to the total fruit cost delivered to either a processing plant or fresh fruit packinghouse. Also, average annual debt payment (principal and interest) may be as high as $\$ 440$ per acre $(\$ 3,750$ average debt per acre@10 percent interest amortized over 20 years) which would reduce total available cash for grove expansion or other investment.

Estimated "delivered-in" costs are shown for processed oranges in Table 4. "Delivered-in" costs include grove care costs (Table 3) plus harvesting, regulatory, and grower assessment costs. The “delivered-in" cost is presented as a cost per acre, per box, and per pound solids. Three possible budget cost scenarios are presented (Refer to Table 11-A): 1) Low Cost Processed Cultural Program;2) Reduced Cost Cultural Program; and 3) Typical/Historical Cultural Program. Scenarios 1 and 2 represent costs of two possible cultural programs directed toward reducing the expenditures for fruit grown primarily for the processed market. The third scenario represents typical costs of grove practices which have been performed for citrus grown for the fresh/processed fruit market. Modified herbicide and/or spray and fertilizer programs account for the reduced costs. NOTE: Before modifying a grove management program to reduce costs, an evaluation of the market program (processed or fresh), yield, and specific cultural problems (nutrition, disease, etc.) for the specific grove site should be made.

\section{HISTORICAL COST TRENDS}

Annual costs and returns for mature, processed Valencia oranges in the Central Florida area have been developed and published the past four years. Estimated cost and return histories for 2000-01 through 2003-04 along with 2004-05 and a five-year average are presented in Table 5. To allow comparisons in current values, these same costs and returns, adjusted to 2005 dollars, are presented in Table 6. 


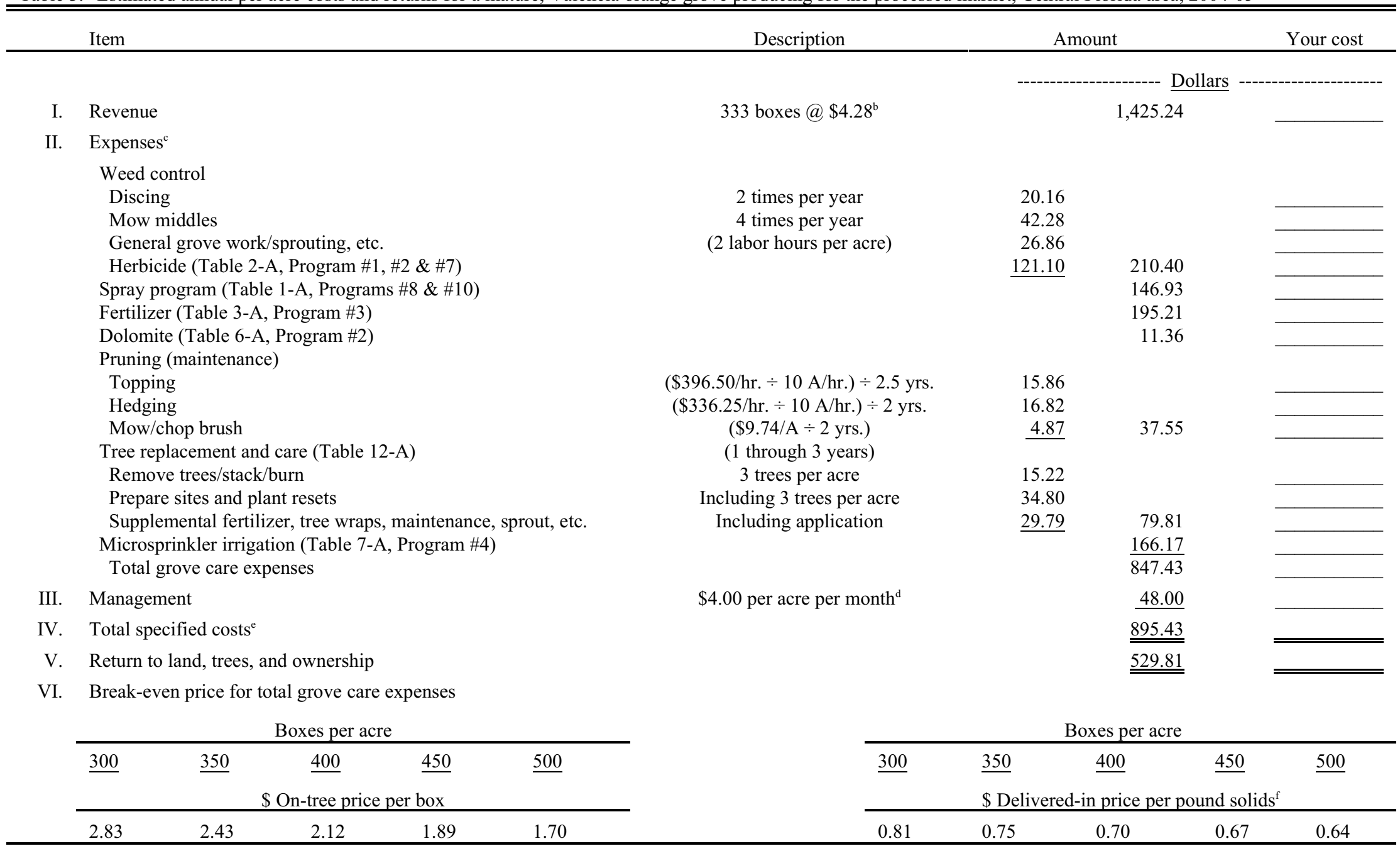

${ }^{2}$ Although the estimated annual per acre grove costs shown in Table 3 are representative for a mature Central Florida Valencia orange grove, the grove care costs for a specific grove site may differ depending upon the grove practices performed; e.g., a Temik application would add $\$ 131.11$ per acre; extensive tree loss due to blight or tristeza would double the tree replacement and care costs; travel and set-up costs may vary due to size of citrus grove and distance from the grove equipment barn.

${ }^{b}$ On-tree price per box is preliminary; assumes price for processed oranges only.

${ }^{c}$ Assumes material custom applied; therefore, a 10 percent handling and supervision charge is added to material cost.

${ }^{d}$ Other methods to estimate a management cost--e.g., $5 \%$ of gross revenue or $10 \%$ of total grove care costs--are used in the industry. Other selected methods will give a different return to land and trees than reported here.

'Other cost items which are not included in the budget are ad valorem taxes and interest on grove investment. In addition to these cost items, overhead and administrative costs, such as water drainage/district taxes, crop insurance, and other grower assessments, can add up to 12 percent to the total grove care costs. These costs vary from grove to grove depending on age, location, and time of purchase or grove establishment.

${ }^{\mathrm{f}}$ Assumes 6.7 pounds solids per box and $\$ 2.348$ pick and haul cost per box (including canker decontamination costs) and Department of Citrus advertising assessment of $\$ 0.165$ per box. 
Table 4. Estimated total delivered-in cost for Central Florida (Ridge) Valencia oranges grown for the processed market under three cultural cost programs, 2004-05

\begin{tabular}{|c|c|c|c|c|c|c|c|c|c|}
\hline \multirow[t]{2}{*}{$\begin{array}{l}\text { Represents a mature }(10+\text { years old }) \\
\text { Central Florida (Ridge) Orange Grove }\end{array}$} & \multicolumn{3}{|c|}{$\begin{array}{c}\text { Processed Valencia Orange } \\
\text { Low Cost } \\
\text { Cultural Program }\end{array}$} & \multicolumn{3}{|c|}{$\begin{array}{l}\text { Processed Valencia Orange } \\
\text { Cultural Program }\end{array}$} & \multicolumn{3}{|c|}{$\begin{array}{c}\text { Fresh/Processed Valencia Orange } \\
\text { Historical Cost } \\
\text { Cultural Program }\end{array}$} \\
\hline & $\$ /$ Acre & $\$ /$ Box & $\$ /$ P.S. & $\$ /$ Acre & $\$ /$ Box & \$/P.S. & $\$ /$ Acre & $\$ /$ Box & $\$ /$ P.S. \\
\hline Total Production/Cultural Costs & $\$ 756.81$ & 2.266 & $\$ 0.3382$ & $\$ 847.43$ & $\$ 2.537$ & $\$ 0.3787$ & $\$ 985.77$ & $\$ 2.951$ & $\$ 0.4405$ \\
\hline Interest on Operating (Cultural) Costs & 20.81 & 0.062 & 0.0093 & 23.30 & 0.070 & 0.0104 & 27.11 & 0.081 & 0.0121 \\
\hline Management Costs & 48.00 & 0.144 & 0.0214 & 48.00 & 0.144 & 0.0214 & 48.00 & 0.144 & 0.0214 \\
\hline \multicolumn{10}{|l|}{ Taxes/Regulatory Costs: } \\
\hline Property Tax and Water Management Tax & 61.87 & 0.185 & 0.0276 & 61.87 & 0.185 & 0.0276 & 61.87 & 0.185 & 0.0276 \\
\hline Canker Decontamination Costs & 5.52 & $\underline{0.017}$ & $\underline{0.0025}$ & 5.52 & $\underline{0.017}$ & $\underline{0.0025}$ & 5.52 & $\underline{0.017}$ & $\underline{0.0025}$ \\
\hline Total Direct Grower Costs & $\$ 893.01$ & $\$ 2.674$ & $\$ 0.3991$ & \$ 986.12 & $\$ 2.952$ & $\$ 0.4407$ & $\$ 1,128.26$ & $\$ 3.378$ & $\$ 0.5042$ \\
\hline Interest on Average Capital Investment Costs & 321.22 & $\underline{0.962}$ & $\$ 0.1435$ & 321.22 & $\underline{0.962}$ & $\underline{0.1435}$ & 321.22 & $\underline{0.962}$ & $\underline{0.1435}$ \\
\hline Total Grower Costs & $\$ 1,214.22$ & $\$ 3.635$ & $\$ 0.5426$ & $\$ 1,307.34$ & $\$ 3.914$ & $\$ 0.5842$ & $\$ 1,449.48$ & $\$ 4.340$ & $\$ 0.6477$ \\
\hline \multicolumn{10}{|l|}{$\begin{array}{l}\text { Harvesting and Assessment Costs: } \\
\text { Pick/Spot Pick, Roadside \& Haul and }\end{array}$} \\
\hline Canker Decontamination Costs & 784.23 & 2.348 & 0.3504 & 784.23 & 2.348 & 0.3504 & 784.23 & 2.348 & 0.3504 \\
\hline DOC Assessment & 55.11 & $\underline{0.165}$ & $\underline{0.0246}$ & 55.11 & $\underline{0.165}$ & $\underline{0.0246}$ & 55.11 & $\underline{0.165}$ & $\underline{0.0246}$ \\
\hline Total Harvesting and Assessment Costs & 839.34 & 2.513 & 0.3751 & 839.34 & 2.513 & 0.3751 & 839.34 & 2.513 & 0.3751 \\
\hline Total Delivered-In Cost & $\$ \underline{\underline{2,053.57}}$ & $\$ \underline{\underline{6.148}}$ & $\$ \underline{\underline{0.9177}}$ & $\$ \underline{\underline{2,146.68}}$ & $\$ \underline{\underline{6.427}}$ & $\$ \underline{\underline{0.9593}}$ & $\$ \underline{\underline{2,288.82}}$ & $\$ \underline{\underline{6.853}}$ & $\$ \underline{\underline{1.0228}}$ \\
\hline $\begin{array}{l}\text { P.S. = Pound Solids } \\
\text { Yield: } 333 \text { boxes/acre @ } 6.7 \text { P.S. per box } \\
112 \text { trees per acre }\end{array}$ & \multicolumn{3}{|c|}{$\begin{array}{l}\text { Refer to cultural program } \\
\text { shown in Table 11-A. } \\
\text { Only summer oil sprays with } \\
\text { oil, copper and Agri-mek \& } \\
\text { Nutritionals. }\end{array}$} & \multicolumn{3}{|c|}{$\begin{array}{l}\text { Refer to cultural program shown } \\
\text { in Table } 3 .\end{array}$} & \multicolumn{3}{|c|}{$\begin{array}{l}\text { Refer to cultural program shown } \\
\text { in Table 11-A. } \\
\text { A Fall Miticide Spray added to the } \\
\text { cultural program shown in Table } 3 .\end{array}$} \\
\hline
\end{tabular}


Table 5.--Estimated annual per acre costs and returns and 5-year average costs and returns for a mature, Valencia orange grove producing citrus for processing in the Central Florida area, 2000-01-2004-05

\begin{tabular}{|c|c|c|c|c|c|c|}
\hline Year & $\begin{array}{c}\text { On-tree } \\
\text { price/box } \\
\end{array}$ & Yield & $\begin{array}{c}\text { Gross } \\
\text { revenue }\end{array}$ & $\begin{array}{c}\text { Total grove } \\
\text { care expenses }\end{array}$ & $\begin{array}{c}\text { Total specified } \\
\text { costs }^{\mathrm{f}} \\
\end{array}$ & $\begin{array}{l}\text { Net return to land, } \\
\text { trees, and ownership }\end{array}$ \\
\hline & & & --------- & \multicolumn{3}{|c|}{$\underline{\text { Dollars }}$} \\
\hline $2000-01$ & $\$ 3.70$ & $436^{\mathrm{d}}$ & $1,613.20$ & $758.85^{\mathrm{e}}$ & 806.85 & 806.35 \\
\hline $2001-02$ & $\$ 4.17$ & 446 & $1,859.82$ & 767.77 & 815.77 & $1,044.05$ \\
\hline $2002-03$ & $\$ 3.80$ & 446 & $1,694.80$ & 777.69 & 825.59 & 869.21 \\
\hline 2003-04 & $\$ 3.67$ & $476^{\mathrm{c}}$ & $1,746.92$ & 774.18 & 822.18 & 924.74 \\
\hline 2004-05 & $\$ 4.28^{\mathrm{b}}$ & $333^{d}$ & $1,425.24$ & 847.43 & 895.43 & 529.81 \\
\hline 5-yr. avg. & $\$ 3.93$ & 427 & $1,678.11$ & 785.19 & 833.19 & 844.92 \\
\hline
\end{tabular}

${ }^{a}$ On-tree prices for processed oranges only as reported by the Florida Agricultural Statistics Service.

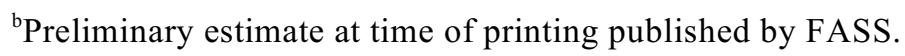

${ }^{\mathrm{c}} \mathrm{Higher}$ per acre yield is due to increased statewide production of Valencia oranges in 2003-04 season.

${ }^{\mathrm{d}}$ The severe drought affected yields for the 2000-01 season and three hurricanes reduced yields $25 \%$ in 2004-05.

${ }^{e}$ Began using two summer oil sprays (one with nutritionals) in budget estimates.

${ }^{\mathrm{f}} \mathrm{A}$ management cost of $\$ 4.00$ per acre per month is included. Fixed costs such as taxes, debt service, and crop insurance are not included. 
Table 6.--Estimated annual per acre costs and returns and 5-year average costs and returns (adjusted to 2005 dollars) for a mature, Valencia orange grove producing citrus for processing in the Central Florida area, 2000-01-2004-05

\begin{tabular}{|c|c|c|c|c|c|c|}
\hline Year & $\begin{array}{c}\text { Inflation } \\
\text { factor index } \\
\end{array}$ & $\begin{array}{l}\text { Adjusted } \\
\text { on-tree } \\
\text { price/box }\end{array}$ & Yield & $\begin{array}{c}\text { Gross } \\
\text { revenue }\end{array}$ & $\begin{array}{c}\text { Total specified } \\
\text { costs }^{b}\end{array}$ & $\begin{array}{l}\text { Net return to land, } \\
\text { trees, and ownership }\end{array}$ \\
\hline & & & & --------. & -1--- Dollars & '-------------------- \\
\hline 2000-01 & 117.9 & $\$ 4.37$ & 436 & $1,905.32$ & 951.28 & 954.04 \\
\hline 2001-02 & 120.7 & $\$ 5.04$ & 446 & $2,247.84$ & 984.64 & $1,263.20$ \\
\hline 2002-03 & 114.6 & $\$ 4.36$ & 446 & $1,944.56$ & 946.13 & 998.43 \\
\hline 2003-04 & 107.9 & $\$ 3.96$ & 476 & $1,884.96$ & 887.14 & 997.82 \\
\hline 2004-05 & 100.0 & $\$ 4.28$ & 333 & $1,425.24$ & 895.43 & 529.81 \\
\hline 5-yr. avg. & - & $\$ 4.41$ & 427 & $1,883.07$ & 932.93 & 950.14 \\
\hline
\end{tabular}

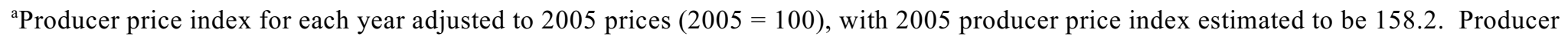
price index for other years are: $2001=134.2 ; 2002=131.1 ; 2003=138.1 ;$ and $2004=146.7$.

${ }^{\mathrm{b}} \mathrm{A}$ management cost of $\$ 4.00$ per acre per month is included. Fixed costs such as taxes, debt service, and crop insurance are not included. (Refer to Table 5.) 


\section{REFERENCES}

1. Preliminary Citrus Summary 2004-05. Florida Agricultural Statistics Service. Florida Agricultural Statistics.

2. Muraro, Ronald P. "A Listing of Estimated Comparative Central Florida (Ridge) Citrus Production Costs Per Acre for 2004-05." Lake Alfred Citrus Research and Education Center (CREC) Report. Lake Alfred, FL: August 2005.

3. _ _ Estimated Cost of Planting and Maintaining a Reset Citrus Tree through Three Years of Age." Lake Alfred CREC Report. Lake Alfred, FL: July 2004.

4. _ . "A Listing of 2005 Custom Rates Reported by Twenty-Five Ridge Citrus Caretakers." Lake Alfred CREC Report. Lake Alfred, FL: July 2005.

5. Savage, Zach. Citrus Yields Per Tree Age. Univ. of Fla. Agr. Ext. Ser. 60-8. Gainesville: 1960.

6. Timmer, L. W. (Ed.). 2005 Florida Citrus Pest Management Guide. Univ. of Fla. Coop. Ext. Svc. SP 43. Gainesville: Jan. 2005. 150 p.

7. Tucker, D. P. H., A. K. Alva, L. K. Jackson, and T. A. Wheaton (Eds.). Nutrition of Florida Citrus Trees. Univ. of Fla. Coop. Ext. Svc. SP 169. Gainesville: 1995. 61 pp. 
ADDENDA: $\quad$ Listing of Grove Care Programs for Central Florida Citrus Production for Both Round Oranges and Grapefruit ${ }^{\mathrm{a}}$

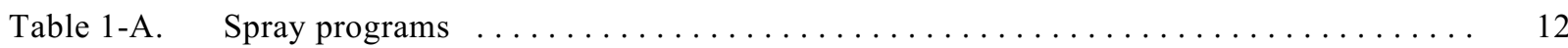

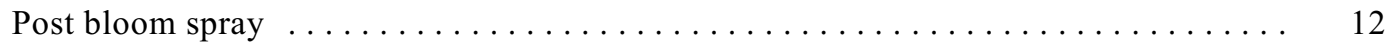

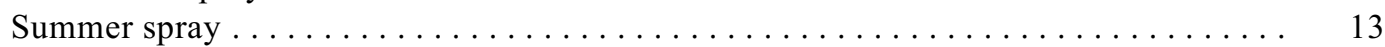

Fall spray $\ldots \ldots \ldots \ldots \ldots \ldots \ldots \ldots \ldots \ldots \ldots \ldots \ldots \ldots \ldots \ldots$

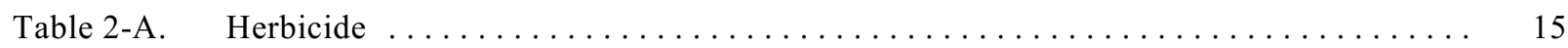

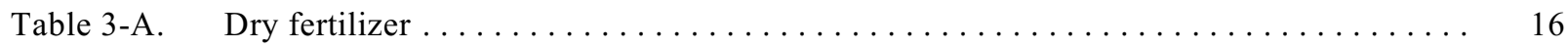

Table 4-A. Liquid fertilizer (Double boom application) $\ldots \ldots \ldots \ldots$

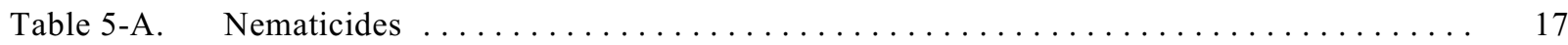

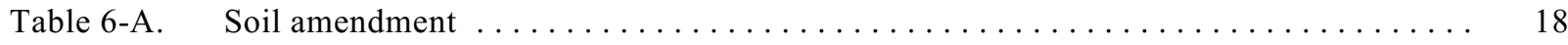

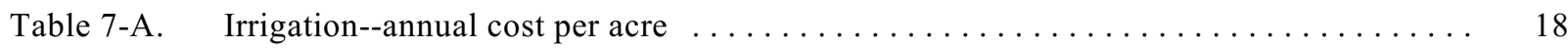

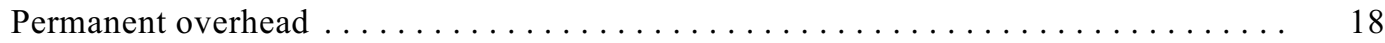

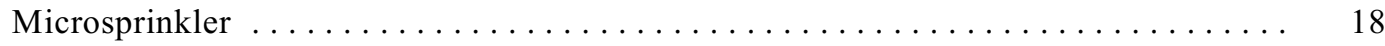

Table 8-A. A listing of 2005 custom rates reported by twenty-five Ridge citrus caretakers . . . . 19

Table 9-A. 2005 summary of average chemical price estimates $\ldots \ldots \ldots 21$

Table 10-A. 2005 summary of average fertilizer price estimates $\ldots \ldots \ldots 23$

Table 11-A. A listing of estimated comparative Central Florida (Ridge) citrus production

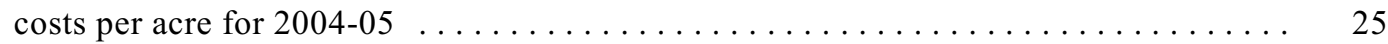

Table 12-A. Estimated cost of planting and maintaining a reset citrus tree through three years

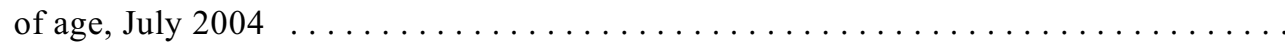

Table 13-A. Estimated average picking, roadsiding and hauling charges for Florida citrus,

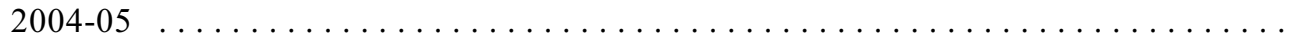

Table 14-A. Estimated average packing charges for Florida citrus, $2004-05 \ldots \ldots 28$

Table 15-A. Historic prices for selected citrus varieties $\ldots \ldots \ldots \ldots \ldots$

Table 16-A. Debt which can be supported per $\$ 1,000.00$ annual payment capacity $\ldots \ldots \ldots$

Abbreviations for important chemicals are:

\begin{tabular}{|c|c|c|}
\hline $\mathrm{B}=$ Boron & $\mathrm{Fe}=$ Iron & Mn $=$ Manganese \\
\hline $\mathrm{Cu}=$ Copper & $\mathrm{Mg}=$ Magnesium & $\mathrm{N}=$ Nitrogen \\
\hline
\end{tabular}

${ }^{\text {a }}$ The costs in the ADDENDA represent a custom managed operation. Therefore, all equipment costs are based upon the average custom rate costs and a 10 percent handling and supervision charge is added to the material cost. 
Table 1-A.--Spray programs

\section{POST BLOOM SPRAY}

\begin{tabular}{|c|c|c|c|c|}
\hline \multirow[t]{7}{*}{ Spray Program \#1 } & $\underline{\text { Materials/Ingredients }}$ & $\begin{array}{l}\text { Amount } \\
\text { /Acre } \\
\end{array}$ & $\underline{\text { Cost/Acre }}$ & $\begin{array}{c}\text { Your } \\
\text { Cost/Acre } \\
\end{array}$ \\
\hline & Oil $97+\%$ & 5 gals & $\$ 12.30$ & \\
\hline & $\mathrm{Cu}(50 \%$ metallic $)$ & $10 \mathrm{lbs}$ & 5.60 & \\
\hline & $\mathrm{Zn}$ & $5 \mathrm{lbs}$ & 4.60 & \\
\hline & $\mathrm{Mn}$ & $10 \mathrm{lbs}$ & 3.60 & \\
\hline & $\begin{array}{l}\text { Ground Application } \\
\text { (PTO driven airblast) }\end{array}$ & 125 gals & $\underline{24.15}$ & \\
\hline & Total per Application & & $\$ \underline{\underline{60.25}}$ & \\
\hline \multirow{7}{*}{$\begin{array}{l}\text { Spray Program \#2 } \\
\text { (Scab/melanose) }\end{array}$} & $\underline{\text { Materials/Ingredients }}$ & $\begin{array}{l}\text { Amount } \\
\text { /Acre } \\
\end{array}$ & $\underline{\text { Cost/Acre }}$ & $\begin{array}{c}\text { Your } \\
\text { Cost/Acre } \\
\end{array}$ \\
\hline & $\mathrm{Cu}(50 \%$ metallic $)$ & $10 \mathrm{lbs}$ & $\$ 15.60$ & \\
\hline & $\mathrm{Zn}$ & $5 \mathrm{lbs}$ & 4.60 & \\
\hline & $\mathrm{Mn}$ & $10 \mathrm{lbs}$ & 3.60 & \\
\hline & Micromite $25 \mathrm{WP}$ & $1.25 \mathrm{lbs}$ & 42.65 & \\
\hline & $\begin{array}{l}\text { Ground Application } \\
\text { (PTO driven airblast) }\end{array}$ & 125 gals & $\underline{24.15}$ & \\
\hline & Total per Application & & $\$ 90.60$ & \\
\hline \multirow[t]{5}{*}{ Spray Program \#3 } & $\underline{\text { Materials/Ingredients }}$ & $\begin{array}{l}\text { Amount } \\
\text { /Acre } \\
\end{array}$ & $\underline{\text { Cost/Acre }}$ & $\begin{array}{c}\text { Your } \\
\text { Cost/Acre } \\
\end{array}$ \\
\hline & $\mathrm{Cu}(50 \%$ metallic $)$ & $15 \mathrm{lbs}$ & $\$ 23.40$ & \\
\hline & Agri-Mek & 10 ozs & 48.60 & \\
\hline & $\begin{array}{l}\text { Ground Application } \\
\text { (engine driven airblast) }\end{array}$ & 250 gals & $\underline{30.30}$ & \\
\hline & Total per Application & & $\$ \underline{\underline{102.30}}$ & \\
\hline \multirow[t]{6}{*}{ Spray Program \#4 } & $\underline{\text { Materials/Ingredients }}$ & $\begin{array}{l}\text { Amount } \\
\text { /Acre }\end{array}$ & $\underline{\text { Cost/Acre }}$ & $\begin{array}{c}\text { Your } \\
\text { Cost/Acre } \\
\end{array}$ \\
\hline & Vendex 50WP & $2 \mathrm{lbs}$ & $\$ 32.70$ & \\
\hline & $\mathrm{Zn}$ & $5 \mathrm{lbs}$ & 4.60 & \\
\hline & $\mathrm{Mn}$ & $10 \mathrm{lbs}$ & 3.60 & \\
\hline & $\begin{array}{l}\text { Ground Application } \\
\text { (PTO driven airblast) }\end{array}$ & 125 gals & $\underline{24.15}$ & \\
\hline & Total per Application & & $\$ \underline{\underline{65.05}}$ & \\
\hline
\end{tabular}


Table 1-A.--Spray programs (cont'd.)

$\underline{\text { POST BLOOM SPRAY (cont'd.) }}$

\begin{tabular}{|c|c|c|c|c|}
\hline Spray Program \#5 & Materials/Ingredients & $\begin{array}{l}\text { Amount } \\
\text { /Acre }\end{array}$ & Cost/Acre & $\begin{array}{c}\text { Your } \\
\text { Cost/Acre } \\
\end{array}$ \\
\hline \multirow[t]{3}{*}{ (Scale insects) } & Lorsban 4EC & $5 \mathrm{pts}$ & $\$ 23.50$ & \\
\hline & $\begin{array}{l}\text { Ground Application } \\
\text { (engine driven airblast) }\end{array}$ & 500 gals & $\underline{32.25}$ & \\
\hline & Total per Application & & $\$ 55.75$ & \\
\hline
\end{tabular}

\section{SUMMER SPRAY}

\begin{tabular}{|c|c|c|c|c|}
\hline \multirow[t]{6}{*}{ Spray Program \#6 } & $\underline{\text { Materials/Ingredients }}$ & $\begin{array}{l}\text { Amount } \\
\text { /Acre } \\
\end{array}$ & $\underline{\text { Cost/Acre }}$ & $\begin{array}{c}\text { Your } \\
\text { Cost/Acre } \\
\end{array}$ \\
\hline & Oil $97+\%$ & 5 gals & $\$ 12.30$ & \\
\hline & $\mathrm{Cu}(50 \%$ material $)$ & $7 \mathrm{lbs}$ & 10.92 & \\
\hline & Micromite & $1.25 \mathrm{lbs}$ & 42.65 & \\
\hline & $\begin{array}{l}\text { Ground Application } \\
\text { (PTO driven airblast) }\end{array}$ & 250 gals & $\underline{30.30}$ & \\
\hline & Total per Application & & $\$ 96.17$ & \\
\hline \multirow[t]{6}{*}{ Spray Program \#7 } & $\underline{\text { Materials/Ingredients }}$ & $\begin{array}{l}\text { Amount } \\
\text { /Acre }\end{array}$ & $\underline{\text { Cost/Acre }}$ & $\begin{array}{c}\text { Your } \\
\text { Cost/Acre } \\
\end{array}$ \\
\hline & Oil $97+\%$ & 5 gals & $\$ 12.30$ & \\
\hline & Agri-Mek & $10 \mathrm{ozs}$ & 48.60 & \\
\hline & $\mathrm{Cu}(50 \%$ material $)$ & $7 \mathrm{lbs}$ & 10.92 & \\
\hline & $\begin{array}{l}\text { Ground Application } \\
\text { (engine driven airblast) }\end{array}$ & 250 gals & 30.30 & \\
\hline & Total per Application & & $\$ \underline{\underline{102.12}}$ & \\
\hline \multirow[t]{6}{*}{ Spray Program \#8 } & $\underline{\text { Materials/Ingredients }}$ & $\begin{array}{l}\text { Amount } \\
\text { /Acre } \\
\end{array}$ & $\underline{\text { Cost/Acre }}$ & $\begin{array}{c}\text { Your } \\
\text { Cost/Acre }\end{array}$ \\
\hline & Oil 97+\% & 5 gals & $\$ 12.30$ & \\
\hline & Micromite & $1.25 \mathrm{lbs}$ & 42.65 & \\
\hline & $\mathrm{Cu}(50 \%$ material $)$ & $7 \mathrm{lbs}$ & 10.92 & \\
\hline & $\begin{array}{l}\text { Ground Application } \\
\text { (PTO driven airblast) }\end{array}$ & 125 gals & $\underline{24.15}$ & \\
\hline & Total per Application & & $\$ 90.02$ & \\
\hline
\end{tabular}


Table 1-A.--Spray programs (cont'd.)

SUMMER SPRAY (cont'd.)

\begin{tabular}{|c|c|c|c|c|}
\hline \multirow[t]{4}{*}{ Spray Program \#9 } & Materials/Ingredients & $\begin{array}{l}\text { Amount } \\
\text { /Acre } \\
\end{array}$ & $\underline{\text { Cost/Acre }}$ & $\begin{array}{c}\text { Your } \\
\text { Cost/Acre } \\
\end{array}$ \\
\hline & Oil 97+\% & 7 gals & $\$ 17.22$ & \\
\hline & $\begin{array}{l}\text { Ground Application } \\
\text { (engine driven airblast) }\end{array}$ & 250 gals & $\underline{30.30}$ & \\
\hline & Total per Application & & $\$ \underline{\underline{47.52}}$ & \\
\hline \multirow[t]{8}{*}{ Spray Program \#10 } & $\underline{\text { Materials/Ingredients }}$ & $\begin{array}{l}\text { Amount } \\
\text { /Acre }\end{array}$ & $\underline{\text { Cost/Acre }}$ & $\begin{array}{c}\text { Your } \\
\text { Cost/Acre } \\
\end{array}$ \\
\hline & $\mathrm{Cu}(50 \%$ metallic $)$ & $7 \mathrm{lbs}$ & $\$ 10.92$ & \\
\hline & Oil 97+\% & 5 gals & 12.30 & \\
\hline & $\mathrm{Zn}$ & $5 \mathrm{lbs}$ & 4.60 & \\
\hline & $\mathrm{Mn}$ & $10 \mathrm{lbs}$ & 3.60 & \\
\hline & $\mathrm{B}$ & $0.25 \mathrm{lbs}$ & 1.34 & \\
\hline & $\begin{array}{l}\text { Ground Application } \\
\quad \text { (PTO driven airblast) }\end{array}$ & 125 gals & $\underline{24.15}$ & \\
\hline & Total per Application & & $\$ \underline{\underline{56.91}}$ & \\
\hline \multicolumn{5}{|l|}{$\underline{\text { FALL SPRAY }}$} \\
\hline \multirow[t]{4}{*}{ Spray Program \#11 } & $\underline{\text { Materials/Ingredients }}$ & $\begin{array}{l}\text { Amount } \\
\text { /Acre } \\
\end{array}$ & $\underline{\text { Cost/Acre }}$ & $\begin{array}{c}\text { Your } \\
\text { Cost/Acre } \\
\end{array}$ \\
\hline & Vendex 50WP & $2 \mathrm{lbs}$ & $\$ 32.70$ & \\
\hline & $\begin{array}{l}\text { Ground Application } \\
\text { (PTO driven airblast) }\end{array}$ & 125 gals & $\underline{24.15}$ & \\
\hline & Total per Application & & $\$ \underline{\underline{56.85}}$ & \\
\hline \multirow[t]{4}{*}{ Spray Program \#12 } & $\underline{\text { Materials/Ingredients }}$ & $\begin{array}{l}\text { Amount } \\
\text { /Acre } \\
\end{array}$ & $\underline{\text { Cost/Acre }}$ & $\begin{array}{c}\text { Your } \\
\text { Cost/Acre } \\
\end{array}$ \\
\hline & Microthiol & $15 \mathrm{lbs}$ & $\$ 11.55$ & \\
\hline & $\begin{array}{l}\text { Ground Application } \\
\text { (PTO driven airblast) }\end{array}$ & 125 gals & $\underline{24.15}$ & \\
\hline & Total per Application & & $\$ 35.70$ & \\
\hline
\end{tabular}


Table 2-A.--Herbicide

\begin{tabular}{|c|c|c|c|c|}
\hline Herbicide Program \#1 & $\underline{\text { Materials }}$ & $\underline{\text { Treated Acre }}$ & Grove Acre $^{\mathrm{a}}$ & $\underline{\text { Grove Acre }}$ \\
\hline (Strip/band) & Solicam 80 DF & $3 \mathrm{lbs}$ & $\$ 23.51$ & \\
\hline & Karmex WP & $4 \mathrm{lbs}$ & 8.52 & \\
\hline & Roundup Ultra Max & 2 qts & 8.02 & \\
\hline & $\begin{array}{l}\text { Ground Application } \\
\text { (1 time) }\end{array}$ & & $\underline{14.00}$ & \\
\hline & Total for 1 Application & & $\$ \underline{\underline{54.05}}$ & \\
\hline Herbicide Program \#2 & $\underline{\text { Materials }}$ & $\begin{array}{c}\text { Amount/ } \\
\text { Treated Acre } \\
\end{array}$ & $\begin{array}{c}\text { Cost/ } \\
\text { Grove Acre }^{\mathrm{a}}\end{array}$ & $\begin{array}{r}\text { Your Cost/ } \\
\text { Grove Acre } \\
\end{array}$ \\
\hline (Strip/band) & Mandate & $2 \mathrm{pts}$ & $\$ 22.85$ & \\
\hline & Direx 4L & 3 qts & 6.84 & \\
\hline & Roundup Ultra Max & 2 qts & 8.02 & \\
\hline & $\begin{array}{l}\text { Ground Application } \\
\text { (1 time) }\end{array}$ & & $\underline{14.00}$ & \\
\hline & Total for 1 Application & & $\$ \underline{\underline{51.71}}$ & \\
\hline Herbicide Program \#3 & $\underline{\text { Materials }}$ & $\begin{array}{c}\text { Amount/ } \\
\text { Treated Acre } \\
\end{array}$ & $\begin{array}{c}\text { Cost/ } \\
\text { Grove Acre }^{\mathrm{a}}\end{array}$ & $\begin{array}{l}\text { Your Cost/ } \\
\text { Grove Acre } \\
\end{array}$ \\
\hline (Strip/band) & Karmex WP & $4 \mathrm{lbs}$ & $\$ 8.52$ & \\
\hline & Roundup Ultra Max & 2 qts & 8.02 & \\
\hline & $\begin{array}{l}\text { Ground Application } \\
\text { (1 time) }\end{array}$ & & $\underline{14.00}$ & \\
\hline & Total for 1 Application & & $\$ \underline{\underline{30.54}}$ & \\
\hline Herbicide Program \#4 & $\underline{\text { Materials }}$ & $\begin{array}{c}\text { Amount/ } \\
\text { Treated Acre } \\
\end{array}$ & $\begin{array}{c}\text { Cost/ } \\
\text { Grove Acre }^{\mathrm{a}}\end{array}$ & $\begin{array}{r}\text { Your Cost/ } \\
\text { Grove Acre }\end{array}$ \\
\hline (Strip/band) & Roundup Ultra Max & $2 \mathrm{qts}$ & $\$ 8.02$ & \\
\hline & Ammonium Sulfate & $17 \mathrm{lbs}$ & 1.49 & \\
\hline & $\begin{array}{l}\text { Ground Application } \\
\text { (1 time) }\end{array}$ & & $\underline{14.00}$ & \\
\hline & Total for 1 Application & & $\$ \underline{\underline{23.51}}$ & \\
\hline Herbicide Program \#5 & $\underline{\text { Materials }}$ & $\begin{array}{c}\text { Amount/ } \\
\text { Treated Acre } \\
\end{array}$ & $\begin{array}{l}\text { Cost/ } \\
\text { Grove Acre }^{\mathrm{a}}\end{array}$ & $\begin{array}{r}\text { Your Cost/ } \\
\text { Grove Acre } \\
\end{array}$ \\
\hline (Strip/band) & Roundup Ultra Max & $2 \mathrm{qts}$ & $\$ 8.02$ & \\
\hline & Princep (Caliber 90) & $4 \mathrm{lbs}$ & 7.24 & \\
\hline & $\begin{array}{l}\text { Ground Application } \\
\text { (1 time) }\end{array}$ & & $\underline{14.00}$ & \\
\hline & Total for 1 Application & & $\$ \underline{\underline{29.26}}$ & \\
\hline
\end{tabular}


Table 2-A.-Herbicide (cont'd.)

\begin{tabular}{|c|c|c|c|c|}
\hline \multirow{6}{*}{$\begin{array}{l}\text { Herbicide Program \#6 } \\
\text { (Strip/band) }\end{array}$} & $\underline{\text { Materials }}$ & $\underline{\text { Treated Acre }}$ & $\underline{\text { Grove Acre }}^{\mathrm{a}}$ & Grove Acre \\
\hline & Direx 4L & $3 \mathrm{qts}$ & $\$ 6.84$ & \\
\hline & Solicam & $3 \mathrm{lbs}$ & 23.51 & \\
\hline & Roundup Ultra Max & 2 qts & 8.02 & \\
\hline & $\begin{array}{l}\text { Ground Application } \\
\text { (1 time) }\end{array}$ & & $\underline{14.00}$ & \\
\hline & Total for 1 Application & & $\$ \underline{\underline{52.37}}$ & \\
\hline \multirow{4}{*}{$\begin{array}{l}\text { Herbicide Program \#7 } \\
\text { (Spot herbicide for } \\
\text { grass/brush regrowth } \\
\text { under trees.) }\end{array}$} & Materials & $\begin{array}{c}\text { Amount/ } \\
\text { Treated Acre }\end{array}$ & $\begin{array}{c}\text { Cost/ } \\
\text { Grove Acre }^{\mathrm{a}}\end{array}$ & $\begin{array}{r}\text { Your Cost/ } \\
\text { Grove Acre }\end{array}$ \\
\hline & Roundup Ultra Max & 2 qts & $\$ 8.02$ & \\
\hline & $\begin{array}{l}\text { Ground Application } \\
\quad(1 \text { time })\end{array}$ & 15 gals & 7.32 & \\
\hline & Total for 1 Application & & $\$ 15.34$ & \\
\hline
\end{tabular}

${ }^{a}$ With respect to herbicide materials, Amount Per Grove Acre does not equal Amount Per Treated Acre shown on the label. Only a strip or band is being treated. In this report, it is assumed that only one-half of a grove surface is being treated.

Table 3-A.--Dry fertilizer

\begin{tabular}{|c|c|c|c|c|}
\hline \multirow{4}{*}{$\begin{array}{l}\text { Program \#1 } \\
(162 \mathrm{lbs} \text { N/Acre })\end{array}$} & $\begin{array}{l}\text { Analysis/Material } \\
\text { Applied }\end{array}$ & $\begin{array}{l}\text { Amount } \\
\text { /Acre } \\
\end{array}$ & $\underline{\text { Cost/Acre }}$ & $\begin{array}{c}\text { Your } \\
\text { Cost/Acre } \\
\end{array}$ \\
\hline & $12-2-12-2.4 \mathrm{MgO}$ & $1350 \mathrm{lbs}$ & $\$ 151.20$ & \\
\hline & Application & 3 times & 26.91 & \\
\hline & Total for 3 Applications & & $\$ 178.11$ & \\
\hline \multirow{4}{*}{$\begin{array}{l}\text { Program \#2 } \\
(180 \mathrm{lbs} \text { N/Acre })\end{array}$} & $\begin{array}{l}\text { Analysis/Material } \\
\text { Applied }\end{array}$ & $\begin{array}{l}\text { Amount } \\
\text { /Acre }\end{array}$ & $\underline{\text { Cost/Acre }}$ & $\begin{array}{c}\text { Your } \\
\text { Cost/Acre } \\
\end{array}$ \\
\hline & $16-0-16-4 \mathrm{MgO}$ & $1125 \mathrm{lbs}$ & $\$ 148.50$ & \\
\hline & Application & 3 times & $\underline{26.91}$ & \\
\hline & Total for 3 Applications & & $\$ \underline{\underline{175.41}}$ & \\
\hline \multirow{4}{*}{$\begin{array}{l}\text { Program \#3 } \\
(204 \mathrm{lbs} \text { N/Acre) }\end{array}$} & $\begin{array}{l}\text { Analysis/Material } \\
\text { Applied }\end{array}$ & $\begin{array}{l}\text { Amount } \\
\text { /Acre }\end{array}$ & $\underline{\text { Cost/Acre }}$ & $\begin{array}{c}\text { Your } \\
\text { Cost/Acre } \\
\end{array}$ \\
\hline & $16-0-16-4 \mathrm{MgO}$ & $1275 \mathrm{lbs}$ & $\$ 168.30$ & \\
\hline & Application & 3 times & 26.91 & \\
\hline & Total for 3 Applications & & $\$ \underline{\underline{195.21}}$ & \\
\hline \multirow{4}{*}{$\begin{array}{l}\text { Program \#4 } \\
(225 \mathrm{lbs} \text { N/Acre })\end{array}$} & $\begin{array}{l}\text { Analysis/Material } \\
\text { Applied }\end{array}$ & $\begin{array}{l}\text { Amount } \\
\text { /Acre }\end{array}$ & $\underline{\text { Cost/Acre }}$ & $\begin{array}{c}\text { Your } \\
\text { Cost/Acre } \\
\end{array}$ \\
\hline & $15-2-15-2.4 \mathrm{MgO}$ & $1500 \mathrm{lbs}$ & $\$ 187.50$ & \\
\hline & Application & 3 times & 26.91 & \\
\hline & Total for 3 Applications & & $\$ 214.41$ & \\
\hline
\end{tabular}


Table 4-A.--Liquid fertilizer (Double boom application)

\begin{tabular}{|c|c|c|c|c|}
\hline Program \#1 & $\begin{array}{l}\text { Analysis/Material } \\
\text { Applied } \\
\end{array}$ & $\begin{array}{l}\text { Amount } \\
\text { /Acre }\end{array}$ & $\underline{\text { Cost/Acre }}$ & $\begin{array}{c}\text { Your } \\
\text { Cost/Acre } \\
\end{array}$ \\
\hline \multirow[t]{3}{*}{ (180 lbs N/Acre) } & $10-0-10$ & $1800 \mathrm{lbs}$ & $\$ 167.40$ & \\
\hline & Double Boom Application & 3 times & $\underline{47.22}$ & \\
\hline & Total for 3 Applications & & $\$ 214.62$ & \\
\hline \multirow{4}{*}{$\begin{array}{l}\text { Program \#2 } \\
(180 \mathrm{lbs} \text { N/Acre })\end{array}$} & $\begin{array}{l}\text { Analysis/Material } \\
\text { Applied } \\
\end{array}$ & $\begin{array}{l}\text { Amount } \\
\text { /Acre } \\
\end{array}$ & $\underline{\text { Cost/Acre }}$ & $\begin{array}{c}\text { Your } \\
\text { Cost/Acre } \\
\end{array}$ \\
\hline & $10-2-10$ & $1800 \mathrm{lbs}$ & $\$ 176.40$ & \\
\hline & Double Boom Application & 3 times & $\underline{47.22}$ & \\
\hline & Total for 3 Applications & & $\$ 223.62$ & \\
\hline \multirow{6}{*}{$\begin{array}{l}\text { Program \#3 } \\
(180 \mathrm{lbs} \text { N/Acre })\end{array}$} & $\begin{array}{l}\text { Analysis/Material } \\
\text { Applied } \\
\end{array}$ & $\begin{array}{l}\text { Amount } \\
\text { /Acre } \\
\end{array}$ & $\underline{\text { Cost/Acre }}$ & $\begin{array}{c}\text { Your } \\
\text { Cost/Acre } \\
\end{array}$ \\
\hline & $10-0-10$ & $1800 \mathrm{lbs}$ & $\$ 167.40$ & \\
\hline & Solicam $80 \mathrm{DF}$ & $3 \mathrm{lbs} *$ & 23.51 & \\
\hline & Karmex WP & 4 lbs* & 8.52 & \\
\hline & Double Boom Application & 3 times & $\underline{47.22}$ & \\
\hline & Total for 3 Applications & & $\$ 246.65$ & \\
\hline
\end{tabular}

Table 5-A.--Nematicides

\begin{tabular}{|c|c|c|c|c|}
\hline \multirow[t]{4}{*}{ Program \#1 } & $\begin{array}{l}\text { Analysis/Material } \\
\text { Applied }\end{array}$ & $\begin{array}{l}\text { Amount } \\
\text { /Acre } \\
\end{array}$ & $\underline{\text { Cost/Acre }}$ & $\begin{array}{c}\text { Your } \\
\text { Cost/Acre } \\
\end{array}$ \\
\hline & Temik 15G & $33 \mathrm{lbs}$ & $\$ 116.16$ & \\
\hline & Application & & 14.95 & \\
\hline & Total per Application & & $\$ \underline{\underline{131.11}}$ & \\
\hline \multirow[t]{4}{*}{ Program \#2 } & $\begin{array}{l}\text { Analysis/Material } \\
\text { Applied } \\
\end{array}$ & $\begin{array}{l}\text { Amount } \\
\text { /Acre }\end{array}$ & Cost/Acre & $\begin{array}{c}\text { Your } \\
\text { Cost/Acre } \\
\end{array}$ \\
\hline & Temik 15G & $17 \mathrm{lbs}$ & $\$ 59.84$ & \\
\hline & Application & & 14.95 & \\
\hline & Total per Application & & $\$ 74.79$ & \\
\hline
\end{tabular}


Table 6-A.--Soil amendment

\begin{tabular}{|c|c|c|c|c|}
\hline \multirow{5}{*}{$\begin{array}{l}\text { Program \#1 } \\
\text { (Every } 3 \text { years) }\end{array}$} & $\begin{array}{l}\text { Analysis/Material } \\
\text { Applied }\end{array}$ & $\begin{array}{l}\text { Amount } \\
\text { /Acre } \\
\end{array}$ & $\underline{\text { Cost/Acre }}$ & $\begin{array}{c}\text { Your } \\
\text { Cost/Acre } \\
\end{array}$ \\
\hline & Dolomite (Delivered) & 1 ton & $\$ 36.05$ & \\
\hline & Application & 1 time & 9.39 & \\
\hline & Total for 1 Application & & $\$ \underline{\underline{45.44}}$ & \\
\hline & (Average $1 / 3$ Ton Applied/Yr) & & $\$ \underline{\underline{15.15}}$ & \\
\hline \multirow{5}{*}{$\begin{array}{l}\text { Program \#2 } \\
\text { (Every } 4 \text { years) }\end{array}$} & $\begin{array}{l}\text { Analysis/Material } \\
\text { Applied }\end{array}$ & $\begin{array}{l}\text { Amount } \\
\text { /Acre } \\
\end{array}$ & $\underline{\text { Cost/Acre }}$ & $\begin{array}{c}\text { Your } \\
\text { Cost/Acre } \\
\end{array}$ \\
\hline & Dolomite (Delivered) & 1 ton & $\$ 36.05$ & \\
\hline & Application & 1 time & 9.39 & \\
\hline & Total for 1 Application & & $\$ \underline{\underline{45.44}}$ & \\
\hline & (Average $1 / 4$ Ton Applied/Yr) & & $\$ \underline{\underline{11.36}}$ & \\
\hline
\end{tabular}

Table 7-A.--Irrigation--annual cost per acre

PERMANENT OVERHEAD

\begin{tabular}{|c|c|c|c|c|}
\hline & $\underline{\text { Program \#1 }}$ & $\begin{array}{c}\text { Your } \\
\text { Cost/Acre } \\
\end{array}$ & $\underline{\text { Program \#2 }}$ & $\begin{array}{c}\text { Your } \\
\text { Cost/Acre } \\
\end{array}$ \\
\hline Operating & $\begin{array}{c}\text { (Electric) } \\
\$ 146.69\end{array}$ & & $\begin{array}{l}\text { (Diesel) } \\
\$ 119.15\end{array}$ & \\
\hline Maintenance of System & 44.98 & & 47.17 & \\
\hline Total Cash Expenses & $\$ 191.67$ & & $\$ 166.33$ & \\
\hline Fixed Depreciation Expense & 55.73 & & 59.54 & \\
\hline Total Cash and Fixed Expenses & $\$ \underline{\underline{247.40}}$ & & $\$ \underline{\underline{222.06}}$ & \\
\hline \multicolumn{5}{|l|}{ MICROSPRINKLER } \\
\hline & $\underline{\text { Program \#3 }}$ & $\begin{array}{c}\begin{array}{c}\text { Your } \\
\text { Cost/Acre }\end{array} \\
\end{array}$ & $\underline{\text { Program \#4 }}$ & $\begin{array}{c}\begin{array}{c}\text { Your } \\
\text { Cost/Acre }\end{array} \\
\end{array}$ \\
\hline Operating & $\begin{array}{l}\text { (Electric) } \\
\$ 70.60^{*}\end{array}$ & & $\begin{array}{l}\text { (Diesel) } \\
\$ 59.44^{*}\end{array}$ & \\
\hline Maintenance of System & 49.08 & & 50.17 & \\
\hline Total Cash Expenses & $\$ 119.68$ & & $\$ 109.61$ & \\
\hline Fixed Depreciation Expense & 52.94 & & 56.56 & \\
\hline Total Cash and Fixed Expenses & $\$ 172.62$ & & $\$ 166.17$ & \\
\hline
\end{tabular}

*Reflects the higher cost of fuel; diesel and electricity. 
Table 8-A.--A listing of 2005 custom rates reported by twenty-five Ridge citrus caretakers

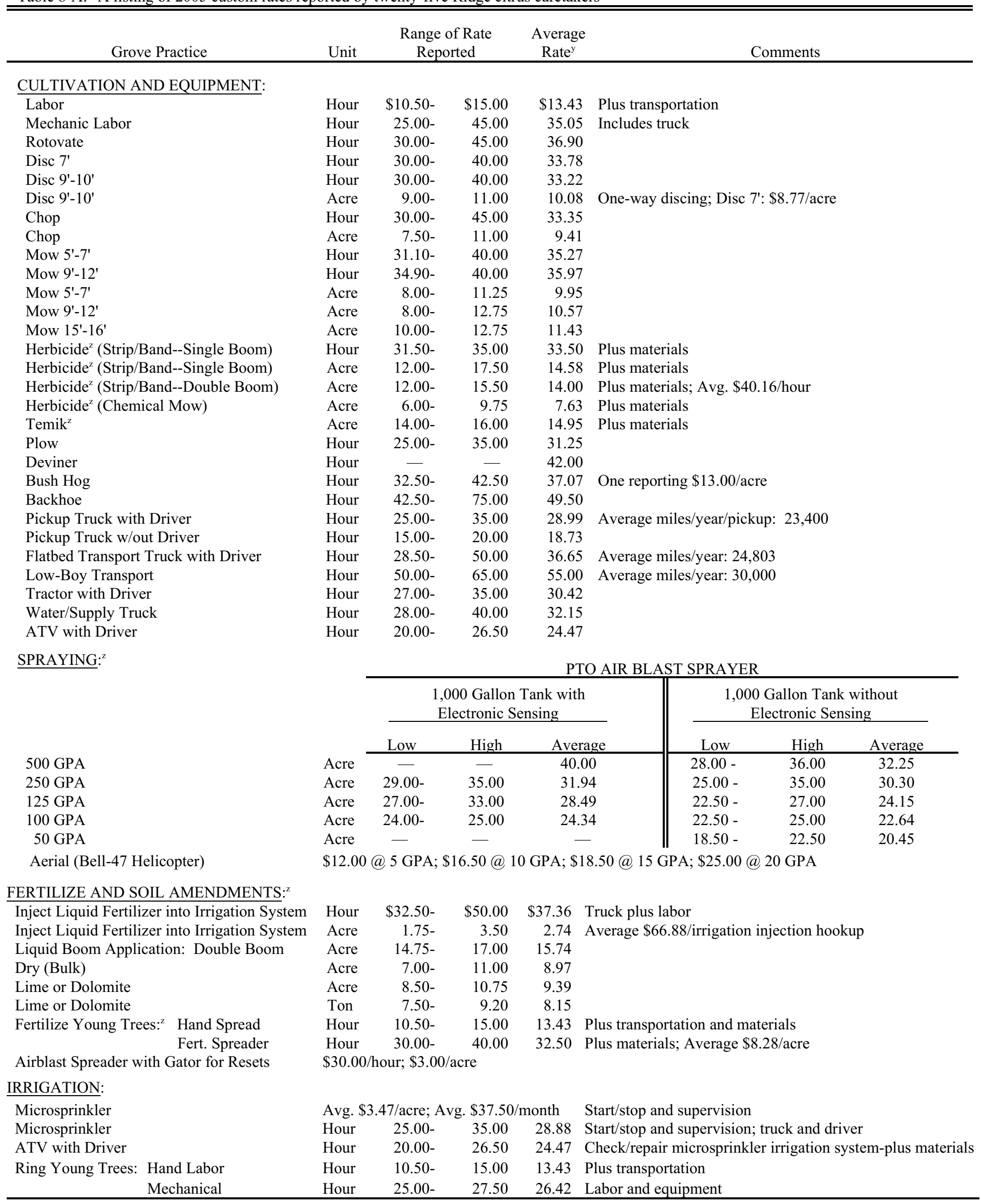


Table 8-A.--A listing of 2005 custom rates reported by twenty-five Ridge citrus caretakers (cont'd.)

\begin{tabular}{|c|c|c|c|c|c|}
\hline Grove Practice & Unit & \multicolumn{2}{|c|}{$\begin{array}{l}\text { Range of Rate } \\
\text { Reported }\end{array}$} & $\begin{array}{c}\text { Average } \\
\text { Rate }^{\mathrm{y}}\end{array}$ & \multirow[t]{2}{*}{ Comments } \\
\hline \multicolumn{5}{|l|}{ REMOVING TREES: } & \\
\hline Tree Shearing (Cutting Tree at Ground Level) & Hour & $\$ 50.00-$ & $\$ 70.00$ & $\$ 61.25$ & \multirow{4}{*}{$\begin{array}{l}\text { Average trees sheared: } 5 \text { to } 20 \text { trees/hour } \\
\text { Average trees removed: } 5 \text { to } 15 \text { trees/hour }\end{array}$} \\
\hline Front-end Loader & Hour & $50.00-$ & 65.00 & 57.63 & \\
\hline Bulldozer & Hour & - & - & 50.00 & \\
\hline Front-end Loader with Tree Spade & Hour & - & - & 65.00 & \\
\hline \multicolumn{6}{|l|}{ PRUNING: } \\
\hline Power Saw with Operator & Hour & $\$ 17.00-$ & $\$ 27.25$ & $\$ 21.08$ & Plus transportation; Average $\$ 7.00 /$ hour without operator \\
\hline Limb Lifter/Tree Skirt Trimmer (Double Sided) & Hour & - & - & 180.00 & \multirow[t]{2}{*}{ Cover $8-12$ acres one pass } \\
\hline Hedging: & & & & & \\
\hline Double Side (Tractor Pulled) & Hour & $85.00-$ & 100.00 & 91.32 & \multirow[t]{3}{*}{ Cover $3-5$ acres/hour } \\
\hline Double Side (Tractor Mounted) & Hour & - & - & 280.00 & \\
\hline Single Side (Self Propelled) & Hour & $65.00-$ & 77.00 & 71.00 & \\
\hline Double Side (Self Propelled) ${ }^{x}$ & Hour & $330.00-$ & 340.00 & 336.25 & Cover $10-25$ acres/hour depending on wood size \\
\hline Double Side (Self Propelled) ${ }^{\mathrm{x}}$ & Hour & $190.00-$ & 250.00 & 216.67 & Cover 4-12 acres/hour depending on wood size \\
\hline \multicolumn{6}{|l|}{ Topping: } \\
\hline Tractor Pulled & Hour & - & - & 280.00 & Cover 5-7 acres/hour \\
\hline Tractor Pulled & Hour & - & - & 105.00 & Cover 1-3 acres/hour \\
\hline Self Propelled & Hour & $375.00-$ & 418.00 & 396.50 & \multirow[t]{2}{*}{ Cover 5-10 acres/hr (Roof Top); 5-20 acres/hr (Flat Top) } \\
\hline Self Propelled & Hour & $190.00-$ & 250.00 & 230.00 & \\
\hline \multicolumn{6}{|l|}{ Removing Brush: } \\
\hline Haul Brush out of Grove & Hour & $32.50-$ & 47.00 & 40.63 & Tractor-trailer/truck; plus 2 people \\
\hline Front-end Loader (Push Brush) & Hour & $50.30-$ & 65.00 & 58.43 & 2-10 acres/hour \\
\hline Chop/Mow Brush & Hour & $31.00-$ & 47.25 & 36.62 & 3-6 acres/hour; Average $\$ 11.63$ /acre \\
\hline \multicolumn{6}{|l|}{ COLD PROTECTION: } \\
\hline Mechanical (Bank and Unbank) & Hour & $\$-$ & $\$-$ & \multicolumn{2}{|l|}{$\$ 28.75$} \\
\hline Install Wraps & Each & $0.35-$ & 0.50 & \multicolumn{2}{|l|}{0.43} \\
\hline Annual Maintenance Costs & Tree & $0.30-$ & 0.50 & \multicolumn{2}{|l|}{0.37} \\
\hline \multicolumn{6}{|l|}{ OTHER CUSTOM RATES: } \\
\hline Plant Resets & Per Tree & $\$ 2.00-$ & $\$ 3.00$ & $\$ 2.55$ & Stake, plant and first watering \\
\hline Solid Set Planting & Per Tree & $1.50-$ & 1.75 & 1.55 & \multirow{2}{*}{ Stake, plant and first watering } \\
\hline Travel/Setup Charge & Hour & - & - & 33.33 & \\
\hline \multicolumn{6}{|l|}{ Grove Management Charge/Month: } \\
\hline Supervising Grove Care Operations & Acre & $2.00-$ & 6.00 & 3.38 & \multirow{3}{*}{ 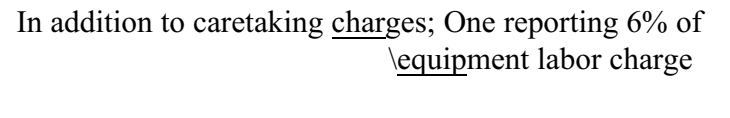 } \\
\hline Handling Fruit Marketing & Box & $0.10-$ & 0.30 & 0.17 & \\
\hline Supervising/Handling Chemicals/Fertilizer & \multicolumn{4}{|c|}{$15 \%$ to $25 \%$ of materials cost } & \\
\hline $\begin{array}{l}\text { Charge for personnel to oversee harvesting } \\
\text { operations and coordinate harvest in } \\
\text { different blocks/groves and keeping of } \\
\text { harvesting labor compliance record. }\end{array}$ & $10 \notin /$ box to & 20غ/box; & verage 14 & /box & $\begin{array}{l}\text { Note: One reporting adding a } 5 \% \text { fuel surcharge on all } \\
\text { billed equipment charges. }\end{array}$ \\
\hline Consulting & $\begin{array}{l}\text { Cultural } \mathrm{N} \\
\text { Financial }\end{array}$ & $\begin{array}{l}\text { anagemen } \\
\text { Analysis } P\end{array}$ & $\begin{array}{l}\text { Horticult } \\
\text { spectus - }\end{array}$ & $\begin{array}{l}\text { ral Evalua } \\
100 / \mathrm{hr} \text { to }\end{array}$ & $\begin{array}{l}\text { tion }-\$ 50 / \mathrm{hr} \text { to } \$ 300 / \mathrm{hr} \\
\$ 300 / \mathrm{hr}\end{array}$ \\
\hline $\begin{array}{l}\text { Total Reported Acreage Provided Grove } \\
\text { Service to: }\end{array}$ & Acre & $800-$ & 15,603 & 3,445 & Total acres reporting: 68,895 \\
\hline
\end{tabular}

${ }^{\text {z }}$ Plus materials. Caretakers reporting rates include labor, tractor and sprayer; supply truck included by most caretakers.

${ }^{y}$ Calculated by dividing the total number of caretakers reporting a grove practice rate into the sum reported. Unless otherwise stated, labor included with all charges.

${ }^{x}$ Low acres is for 2 years regrowth hedging; high acres is for annual maintenance hedging.

Source: Ronald P. Muraro, Extension Farm Management Economist, Lake Alfred CREC, July 2005. 
Table 9-A.--2005 summary of average chemical price estimates

\begin{tabular}{|c|c|c|c|c|}
\hline \multicolumn{2}{|l|}{ Item } & Unit & $\begin{array}{l}\text { Average } \\
\text { Price }\end{array}$ & $\begin{array}{c}\text { Your Price } \\
(2005)\end{array}$ \\
\hline \multirow{16}{*}{ Fungicides: } & Abound EC & gal. & 218.12 & \\
\hline & Aliette 80WP & lb. & 11.59 & \\
\hline & Basic Copper Sulfate & lb. & 1.40 & \\
\hline & Copper (Kocide 101) & lb. & 1.80 & \\
\hline & Copper (Kocide 2000) & lb. & 2.33 & \\
\hline & Copper (Champ II Flowable) & gal. & 22.55 & \\
\hline & Cuprofix Disperss & lb. & 1.75 & \\
\hline & Nu-Cop 50 DF & lb. & 1.88 & \\
\hline & Enable & gal. & 57.55 & \\
\hline & Gem 25 & 40 ozs. & 120.59 & \\
\hline & Headline EC & gal. & 206.13 & \\
\hline & Oil -435 or 455 & gal. & 2.21 & \\
\hline & Oil - 470 (Bio-lever) & gal. & 2.46 & \\
\hline & Ridomil Gold EC & gal. & 649.15 & \\
\hline & Safe-T-Oil & gal. & 3.15 & \\
\hline & Topsin & lb. & 14.08 & \\
\hline \multicolumn{5}{|c|}{ Insecticides/Nematicides: } \\
\hline & Admire $2 \mathrm{~F}$ & gal. & 520.28 & \\
\hline & Agri-Mek (0.15EC) & gal. & 563.52 & \\
\hline & Award Fire Ant Bait & lb. & 9.01 & \\
\hline & Bio-Vector & gal. & 412.50 & \\
\hline & Carbaryl 4L & gal. & 27.25 & \\
\hline & Carbaryl 80S & lb. & 4.47 & \\
\hline & Chlorpyrifos $4 \mathrm{E}$ & gal. & 57.26 & \\
\hline & Danitol & gal. & 147.58 & \\
\hline & Guthion 2L & gal. & 32.48 & \\
\hline & Guthion 50WP & lb. & 10.07 & \\
\hline & Imidan 70W (Diaprepes) & lb. & 8.25 & \\
\hline & Lorsban 4EC & gal. & 34.15 & \\
\hline & Lorsban $15 \mathrm{G}$ & lb. & 1.72 & \\
\hline & Malathion $5 \mathrm{EC}$ & gal. & 25.18 & \\
\hline & Micromite $80 \mathrm{WG}$ & gal. & 87.95 & \\
\hline & Microthiol & lb. & 0.70 & \\
\hline & Nexter 75WP & lb. & 89.56 & \\
\hline & Provado 1.6 F (nursery) & gal. & 417.75 & \\
\hline & Sevin $80 \mathrm{~S}$ & lb. & 5.17 & \\
\hline & Sevin XLR & gal. & 30.96 & \\
\hline & Spintor $2 \mathrm{~S} \mathrm{C}$ & gal. & 492.50 & \\
\hline & Sulphur 6F & gal. & 4.00 & \\
\hline & Temik 15G & lb. & 3.20 & \\
\hline & Vendex $50 \mathrm{~W}$ & lb. & 14.86 & \\
\hline & Vydate & gal. & 56.28 & \\
\hline
\end{tabular}


Table 9-A.--2005 summary of average chemical price estimates (cont'd.)

\begin{tabular}{|c|c|c|c|c|}
\hline \multicolumn{2}{|l|}{ Item } & \multirow{2}{*}{$\frac{\text { Unit }}{\text { gal. }}$} & \multirow{2}{*}{$\begin{array}{c}\begin{array}{c}\text { Average } \\
\text { Price }\end{array} \\
48.39\end{array}$} & \multirow[t]{2}{*}{$\begin{array}{c}\text { Your Price } \\
(2005) \\
\end{array}$} \\
\hline Herbicides: & Aqua Master & & & \\
\hline & Diuron 4L & gal. & 16.04 & \\
\hline & Direx 4L & gal. & 16.50 & \\
\hline & Direx $80 \mathrm{DF}$ & lb. & 3.87 & \\
\hline & Fusilade DX 2E & gal. & 131.14 & \\
\hline \multicolumn{5}{|c|}{ Glyphosate: } \\
\hline & Glyphomax Plus & gal. & 18.22 & \\
\hline & Roundup (Original) & gal. & 23.60 & \\
\hline & Roundup - Ultra Max & gal. & 29.12 & \\
\hline & Roundup Weather Max & gal. & 50.16 & \\
\hline & Roundup Original Max & gal. & 43.50 & \\
\hline & Touchdown & gal. & 37.05 & \\
\hline & Gramoxone E (Paraquat) & gal. & 37.53 & \\
\hline & Hyvar X 80 WP & lb. & 18.93 & \\
\hline & Karmex 80 DF & lb. & 3.87 & \\
\hline & Krovar I & lb. & 11.38 & \\
\hline & Landmaster II & gal. & 18.66 & \\
\hline & Mandate 2E & gal. & 166.09 & \\
\hline & Pendimax & gal. & 24.37 & \\
\hline & Poast Plus 1.0 EC & gal. & 52.50 & \\
\hline & Princep (Caliber 90) & lb. & 3.29 & \\
\hline & Princep 4L & gal. & 14.51 & \\
\hline & Prowl & gal. & 22.12 & \\
\hline & Simazine $90 \mathrm{DF}$ & lb. & 2.80 & \\
\hline & Simazine 4L & gal. & 13.66 & \\
\hline & Solicam 80 DF & lb. & 14.24 & \\
\hline & Simtrol & & 19.00 & \\
\hline & Surflan & gal. & 81.64 & \\
\hline \multicolumn{5}{|c|}{ Growth Regulators: } \\
\hline & Citrus Fix & gal. & 494.00 & \\
\hline & Pro-Gibb 3.91\% & 20 oz. bottle & 33.16 & \\
\hline & Tree-Hold & gal. & 79.17 & \\
\hline \multicolumn{5}{|c|}{ Other Spray Materials: } \\
\hline & Borates $(15 \%)$ & lb. & 0.70 & \\
\hline & Manganese (32\%) & lb. & 0.32 & \\
\hline & Zinc $(78 \%)$ & lb. & 0.83 & \\
\hline & Adjuvant (Surfactant) & gal. & 23.59 & \\
\hline
\end{tabular}

SOURCE: Ronald P. Muraro, Extension Farm Management Economist, University of Florida, IFAS, CREC, Lake Alfred, Florida, August 2005. 
Table 10-A.--2005 summary of average fertilizer price estimates

\begin{tabular}{|c|c|c|c|}
\hline Item & Unit & $\begin{array}{c}\text { Average } \\
\text { Price } \\
\end{array}$ & $\begin{array}{c}\text { Your Price } \\
(2005) \\
\end{array}$ \\
\hline \multicolumn{4}{|l|}{ 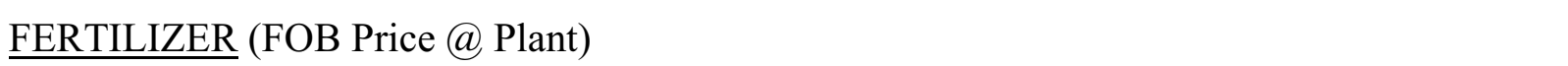 } \\
\hline & & $\$$ & \\
\hline \multicolumn{4}{|l|}{$\underline{\text { Dry Mix (Bulk) }}$} \\
\hline $17-0-17-3_{\mathrm{Mg}}$ & ton & 238.82 & \\
\hline $17-4-17-2.4_{\mathrm{Mg}}$ & ton & 243.35 & \\
\hline $16-0-16$ & ton & 218.35 & \\
\hline $16-0-16-4_{\mathrm{Mg}}$ & ton & 239.49 & \\
\hline $16-2-16-3_{\mathrm{Mg}}$ & ton & 240.45 & \\
\hline $15-2-15-2.4_{\mathrm{Mg}}$ & ton & 224.47 & \\
\hline $12-2-12-2.4_{\mathrm{Mg}}$ & ton & 201.02 & \\
\hline $8-8-8 \mathrm{w} / \mathrm{minors} *$ & ton & 182.90 & \\
\hline $8-4-8 \mathrm{w} /$ minors* & ton & 170.29 & \\
\hline $8-2-8 \mathrm{w} /$ minors $*$ & ton & 162.12 & \\
\hline $6-6-6 \mathrm{w} / \mathrm{minors} *$ & ton & 159.99 & \\
\hline \multicolumn{4}{|l|}{$\underline{\text { Liquid Mix (Bulk) }}$} \\
\hline $8-2-8$ & ton & 151.53 & \\
\hline $8-4-8$ & ton & 159.73 & \\
\hline $9-3-9$ & ton & 166.33 & \\
\hline $9-4-9$ & ton & 172.47 & \\
\hline $10-0-10$ & ton & 166.62 & \\
\hline $10-2-10$ & ton & 176.25 & \\
\hline $12-0-6$ & ton & 166.89 & \\
\hline $12-3-6$ & ton & 180.25 & \\
\hline 7-0-0-6 (Magnesium Nitrate) & ton & 218.00 & \\
\hline
\end{tabular}

*With organic nitrogen, the price averaged $25 \%$ higher. 
Table 10-A.--2005 summary of average fertilizer price estimates (cont'd.)

\begin{tabular}{|c|c|c|c|}
\hline Item & Unit & $\begin{array}{c}\text { Average } \\
\text { Price }\end{array}$ & $\begin{array}{c}\text { Your Price } \\
(2005) \\
\end{array}$ \\
\hline \multicolumn{4}{|l|}{ Other Fertilizer Materials (Bulk) } \\
\hline Ammonium Nitrate (21\% N Liquid) & ton & 179.88 & \\
\hline Ammonium Nitrate (33.5\% N Dry) & ton & 259.38 & \\
\hline Ammonium Sulfate $(21 \% \mathrm{~N})$ & ton & 152.94 & \\
\hline Calcium Nitrate $(19 \% \mathrm{Ca}, 15.5 \% \mathrm{~N})$ & ton & 288.13 & \\
\hline Dolomite (at mine-- $49 \% \mathrm{CaCO}_{3}, 36 \% \mathrm{MgCO}_{3}$ ) & ton & 19.75 & \\
\hline Muriate of Potash $\left(60 \% \mathrm{~K}_{2} \mathrm{O}\right)$ & ton & 242.29 & \\
\hline Potassium Nitrate $\left(14 \% \mathrm{~N} ; 46 \% \mathrm{~K}_{2} \mathrm{O}\right)$ & ton & 453.57 & \\
\hline Sul-Po-Mag (SPM--21.9\% $\left.\mathrm{K}_{2} \mathrm{O}\right)$ & ton & 202.43 & \\
\hline Super Phosphate $\left(20 \% \mathrm{P}_{2} \mathrm{O}_{5}\right)$ & ton & 214.25 & \\
\hline Triple Superphosphate $\left(48 \% \mathrm{P}_{2} \mathrm{O}_{5}\right)$ & ton & 242.92 & \\
\hline Average Delivery Cost & ton & 14.32 & \\
\hline \multicolumn{4}{|l|}{$\underline{\text { Foliar Macronutrients }}$} \\
\hline Phos Might 0-22-20 & gal. & 24.29 & \\
\hline Nutriphite Magnum 2-40-16 & gal. & 35.00 & \\
\hline MKP (0-52-34) (Mono-Potassium Phosphate) & lb. & 0.80 & \\
\hline RSA ActaPhos 0-28-25 & gal. & 18.00 & \\
\hline Peter's 20-20-20 Foliar & lb. & 0.54 & \\
\hline $\mathrm{MZF}$ & gal. & 6.53 & \\
\hline \multicolumn{4}{|l|}{$\underline{\text { Slow Release Nitrogen (SRN) }}$} \\
\hline \multicolumn{4}{|l|}{$\underline{\text { CitriBlen }}$} \\
\hline $15-3-19$ & ton & 245.15 & \\
\hline $17-5-12$ & ton & 237.50 & \\
\hline $18-6-11$ & ton & 243.80 & \\
\hline Sulfur Coated Urea (SCU) & ton & 586.80 & \\
\hline Agriform 20-10-5 (500 tablets/box) & box & 40.00 & \\
\hline
\end{tabular}

SOURCE: Ronald P. Muraro, Extension Farm Management Economist, University of Florida, IFAS, CREC, Lake Alfred, Florida, August 2005. 


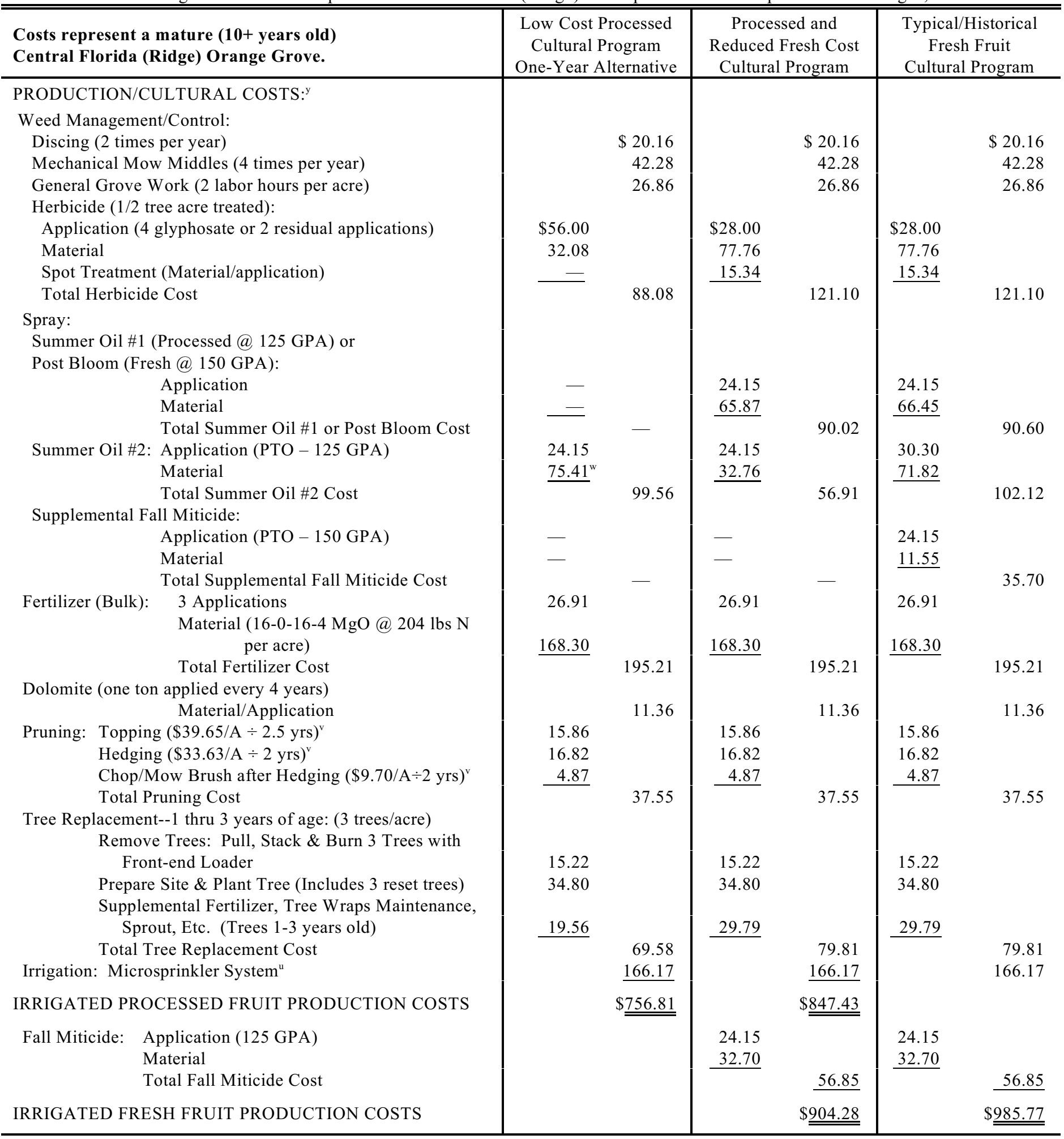

${ }^{\mathrm{z}}$ The listed estimated comparative costs are for the example grove situation described in the Economic Information Report Series entitled: "Budgeting Costs and Returns for Central Florida Citrus Production" and may not represent your particular grove situation in Central Florida.

SOURCE: Ronald P. Muraro, University of Florida-IFAS, Citrus Research and Education Center, Lake Alfred, FL, August 2005. 
Table 12-A.--Estimated cost of planting and maintaining a reset citrus tree through three years of age, Central Florida area, August 2005

\begin{tabular}{|c|c|c|c|c|c|}
\hline & \multicolumn{5}{|c|}{ Resets/Replacement Trees Per Acre } \\
\hline & $1-2$ & $3-5$ & $6-10$ & $11-25$ & $26+$ \\
\hline & \multicolumn{5}{|c|}{-------------- \$ Cost Per Tree ----------- } \\
\hline Tree Removal & 6.67 & 5.34 & 4.45 & 3.56 & 2.67 \\
\hline \multicolumn{6}{|l|}{$\underline{\text { Plant Reset Tree }}$} \\
\hline Tree Cost (Container Tree) & 4.50 & 4.50 & 4.35 & 4.35 & 4.35 \\
\hline Plant Tree and First Watering (Custom Charge) & 2.93 & $\underline{2.55}$ & $\underline{2.17}$ & 1.84 & 1.57 \\
\hline Total Planting Costs & 7.43 & 7.05 & 6.52 & 6.19 & 5.92 \\
\hline
\end{tabular}

$\underline{\text { Site Preparation }}^{\mathrm{a}}$

Disk Tree Site

$\begin{array}{lllll}2.65 & 2.31 & 1.96 & 1.67 & 1.42 \\ \underline{2.65} & \underline{2.31} & \underline{1.97} & \underline{1.67} & \underline{1.42} \\ 5.30 & 4.62 & 3.93 & 3.34 & 2.84\end{array}$

Total Site Preparation

$\begin{array}{lllll}12.73 & 11.67 & 10.45 & 9.53 \quad 8.76\end{array}$

Total Planting and Site Preparation Costs

$=====================$

\begin{tabular}{lrrrrrr} 
Supplemental Maintenance & Year\#1 & 4.13 & 3.82 & 3.59 & 3.39 & 3.19 \\
(Trees 1-3 years old) & Year \#2 & 3.79 & 3.39 & 2.96 & 2.59 & 2.27 \\
$\begin{array}{l}\text { (Fertilizer, Tree Wraps, Sprout, etc.) } \\
\quad \text { Year \#3 }\end{array}$ & $\underline{3.07}$ & $\underline{2.73}$ & $\underline{2.34}$ & $\underline{2.01}$ & $\underline{1.73}$ \\
$\quad$ Total Supplemental Maintenance Costs & 10.99 & 9.94 & 8.89 & 7.99 & 7.19 \\
$==================================================$ \\
Summary of Tree Replacement Costs & 1 & 3 & 6 & 6 & 6 \\
\hline Tree Removal Costs & 6.67 & 5.34 & 4.45 & 3.56 & 2.67 \\
Planting and Site Preparation Costs & 12.73 & 11.67 & 10.45 & 9.53 & 8.76 \\
Supplemental Maintenance Costs (Years 1 thru 3) & $\underline{10.99}$ & $\underline{9.94}$ & $\underline{8.89}$ & $\underline{7.99}$ & $\underline{7.19}$ \\
$\quad$ & $\underline{\underline{30.39}}$ & $\underline{\underline{26.95}}$ & $\underline{\underline{23.79}}$ & $\underline{\underline{21.08}}$ & $\underline{\underline{18.62}}$ \\
\hline
\end{tabular}

${ }^{\text {a}}$ Fumigate planting site would cost approximately $\$ 2.50$ per tree.

Source: Ronald P. Muraro, Farm Management Economist, CREC, Lake Alfred, FL, August 2005. 
Table 13-A.-- Estimated average picking, roadsiding and hauling charges for Florida citrus, 2004-05

\begin{tabular}{|c|c|c|c|c|c|c|}
\hline & \multicolumn{3}{|c|}{ Fresh Fruit } & \multicolumn{3}{|c|}{ Processed Fruit } \\
\hline & & Range & Average & & Range & Average \\
\hline & & $\$ /$ Box & $\$ /$ Box & & $\$ /$ Box & $\$ /$ Box \\
\hline \multicolumn{7}{|l|}{ Picking Charges: } \\
\hline Early and Mid-Season Oranges & 0.70 & $-\quad 1.75$ & 0.954 & 0.65 & $-\quad 1.05$ & 0.829 \\
\hline Valencia Oranges & 0.70 & $-\quad 1.25$ & 0.938 & 0.65 & -1.25 & 0.870 \\
\hline Pink/Red Grapefruit & 0.60 & $-\quad 1.25$ & 0.739 & 0.55 & -1.25 & 0.669 \\
\hline White/Marsh Grapefruit & 0.60 & $-\quad 1.25$ & 0.744 & 0.55 & $-\quad 1.25$ & 0.667 \\
\hline Temples/Tangelos & 0.85 & $-\quad 1.65$ & 1.163 & 0.80 & $-\quad 1.50$ & 1.043 \\
\hline Tangerines & 1.25 & $-\quad 2.00$ & 1.529 & 1.00 & -1.70 & 1.204 \\
\hline \multirow[t]{4}{*}{ Add for Spot Picking } & 0.10 & $-\quad 0.50$ & 0.314 & & - & - \\
\hline & \multicolumn{3}{|c|}{ Fresh Fruit } & \multicolumn{3}{|c|}{ Processed Fruit } \\
\hline & \multicolumn{2}{|c|}{ Range } & Average & \multicolumn{2}{|r|}{ Range } & Average \\
\hline & \multicolumn{2}{|c|}{$\$ /$ Box } & $\$ /$ Box & \multicolumn{2}{|r|}{$\$ /$ Box } & $\$ /$ Box \\
\hline \multicolumn{7}{|l|}{ Roadsiding Charges: } \\
\hline Early and Mid-Season Oranges & 0.60 & $-\quad 1.15$ & 0.895 & 0.65 & $-\quad 1.17$ & 0.817 \\
\hline Valencia Oranges & 0.67 & $-\quad 1.12$ & 0.899 & 0.65 & $-\quad 1.17$ & 0.836 \\
\hline Pink/Red Grapefruit & 0.65 & -1.03 & 0.840 & 0.65 & $-\quad 1.20$ & 0.796 \\
\hline White/Marsh Grapefruit & 0.65 & $-\quad 1.03$ & 0.854 & 0.65 & $-\quad 1.20$ & 0.789 \\
\hline Temples/Tangelos & 0.70 & $-\quad 1.35$ & 1.003 & 0.75 & $-\quad 1.23$ & 0.890 \\
\hline \multirow[t]{4}{*}{ Tangerines } & 0.75 & $-\quad 1.35$ & 1.095 & 0.85 & $-\quad 1.70$ & 1.054 \\
\hline & \multicolumn{3}{|c|}{ Fresh Fruit } & \multicolumn{3}{|c|}{ Processed Fruit } \\
\hline & \multicolumn{3}{|c|}{ All Varieties } & \multicolumn{3}{|c|}{ All Varieties } \\
\hline & \multicolumn{3}{|c|}{$\$ /$ Box } & \multicolumn{3}{|c|}{$\$ /$ Box } \\
\hline \multicolumn{7}{|l|}{ Hauling Charges: } \\
\hline 0 - 30 miles & \multicolumn{3}{|c|}{0.417} & \multicolumn{3}{|c|}{0.393} \\
\hline $31-50$ miles & \multicolumn{3}{|c|}{0.512} & \multicolumn{3}{|c|}{0.464} \\
\hline $51-80$ miles & \multicolumn{3}{|c|}{0.573} & \multicolumn{3}{|c|}{0.515} \\
\hline $81-100$ miles & \multicolumn{3}{|c|}{0.640} & \multicolumn{3}{|c|}{0.632} \\
\hline $100+$ miles & \multicolumn{3}{|c|}{0.746} & \multicolumn{3}{|c|}{0.728} \\
\hline
\end{tabular}


Table 14-A.--Estimated Average Packing Charges for Florida Citrus, 2004-05 a

\begin{tabular}{|c|c|c|c|c|c|}
\hline & $\begin{array}{l}\text { Domestic } \\
\text { Grapefruit }\end{array}$ & $\begin{array}{c}\text { Export } \\
\text { Grapefruit }\end{array}$ & Oranges & $\begin{array}{l}\text { Temples/ } \\
\text { Tangelos }\end{array}$ & Tangerines \\
\hline & & & \$/Carton & ----------. & ------- \\
\hline \multirow[t]{2}{*}{ Total Packing Charge $^{\mathrm{b}}$} & 3.835 & 4.245 & 4.192 & 4.495 & 5.056 \\
\hline & ------ & 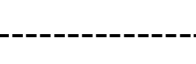 & \$/Box & ----- & ------ \\
\hline Drenching Charge & 0.178 & 0.178 & 0.186 & 0.186 & 0.186 \\
\hline $\begin{array}{l}\text { Packinghouse Elimination } \\
\text { Charges }\end{array}$ & 0.594 & 0.594 & 0.571 & 0.571 & 0.571 \\
\hline $\begin{array}{l}\text { Hauling Charges for } \\
\text { Eliminations }\end{array}$ & 0.425 & 0.425 & 0.410 & 0.410 & 0.410 \\
\hline
\end{tabular}

${ }^{\text {a }}$ Packing charges represents a total of nine citrus packinghouses from both the Indian River and Interior production regions.

${ }^{\mathrm{b}}$ Total Packing Charge includes the following items:

1. Materials including mesh/plastic bags, labels/Price Lookup Codes (PLUs), etc.

2. Includes supervisor/foreman labor, grading, palletizing, shipping and general labor. Includes payroll taxes, workers' compensation, ground insurance, etc.

3. Other direct packing costs include: fruit treating; power, lights and water; repairs maintenance; miscellaneous supplies; etc.

4. Indirect packing costs include such items as: insurance-fire and casualty; taxes and licenses; depreciation and rent.

5. General and Administrative (G\&A) costs include: office personnel (payroll taxes, w/comp); packinghouse and general manager; office supplies; telephone; etc.

6. Selling Expenses which include sales salaries, travel, telephone and telegraph and brokerage fees.

7. Special assessments include such items as: advertising taxes; inspection fees; a Florida Citrus Packers tax; and a Citrus Administrative Committee (CAC) tax.

SOURCE: Ronald P. Muraro, University of Florida-IFAS, Citrus Research and Education Center, Lake Alfred, FL, September 2005. 
Table 15-A.--Historic prices ${ }^{\mathrm{a}}$ for selected citrus varieties

\begin{tabular}{|c|c|c|c|c|c|c|c|}
\hline \multirow[b]{3}{*}{ Crop year } & \multicolumn{7}{|c|}{ Variety } \\
\hline & \multirow{2}{*}{$\begin{array}{c}\text { Early }^{\mathrm{b}} \text { and } \\
\text { mid }^{\mathrm{c}} \text {-season } \\
\text { oranges }\end{array}$} & \multirow{2}{*}{$\begin{array}{c}\text { Late season } \\
\text { oranges }^{\mathrm{d}}\end{array}$} & \multirow{2}{*}{$\begin{array}{l}\text { Temple } \\
\text { oranges }\end{array}$} & \multirow{2}{*}{$\begin{array}{c}\text { All } \\
\text { Tangerines } \\
\end{array}$} & \multirow[b]{2}{*}{ Tangelos } & \multicolumn{2}{|c|}{ Seedless grapefruit ${ }^{\mathrm{e}}$} \\
\hline & & & & & & (white) & (colored) \\
\hline $1961-62$ & $\$ 1.93$ & $\$ 1.81$ & $\$ 2.17$ & $\$ 2.04$ & $\$ 3.36$ & $\$ 0.68$ & $\$ 0.86$ \\
\hline $1962-63$ & 2.17 & 3.50 & 3.09 & 3.02 & 4.66 & 1.29 & 1.81 \\
\hline $1963-64$ & 4.43 & 4.45 & 4.45 & 3.18 & 4.83 & 2.24 & 2.54 \\
\hline $1964-65$ & 2.57 & 2.28 & 2.77 & 2.68 & 4.00 & 1.51 & 1.82 \\
\hline $1965-66$ & 1.44 & 1.79 & 1.80 & 2.14 & 2.85 & 1.39 & 1.64 \\
\hline $1966-67$ & 0.81 & 1.08 & 0.88 & 1.06 & 1.64 & 0.73 & 0.94 \\
\hline $1967-68$ & 1.86 & 2.28 & 2.79 & 4.29 & 3.22 & 2.05 & 2.48 \\
\hline $1968-69$ & 1.56 & 1.83 & 2.22 & 2.55 & 2.47 & 0.98 & 1.15 \\
\hline $1969-70$ & 1.15 & 1.13 & 1.47 & 2.23 & 1.13 & 1.72 & 1.92 \\
\hline $1970-71$ & 1.10 & 1.91 & 1.91 & 1.88 & 1.04 & 1.89 & 2.15 \\
\hline $1971-72$ & 1.98 & 2.11 & 1.95 & 2.97 & 1.69 & 2.27 & 2.69 \\
\hline $1972-73$ & 1.43 & 1.71 & 1.95 & 2.37 & 1.39 & 2.06 & 2.53 \\
\hline $1973-74$ & 1.38 & 1.59 & 1.64 & 2.82 & 1.25 & 1.58 & 2.12 \\
\hline $1974-75$ & 1.46 & 1.82 & 1.68 & 3.05 & 1.45 & 1.55 & 2.59 \\
\hline $1975-76$ & 1.69 & 1.88 & 1.79 & 3.02 & 1.42 & 1.29 & 2.23 \\
\hline $1976-77$ & 1.89 & 2.63 & 2.16 & 3.29 & 1.42 & 1.49 & 2.04 \\
\hline $1977-78$ & 3.90 & 4.40 & 3.92 & 4.79 & 3.29 & 1.47 & 2.09 \\
\hline $1978-79$ & 4.44 & 4.95 & 4.89 & 4.99 & 3.90 & 2.21 & 3.13 \\
\hline $1979-80$ & 3.59 & 3.89 & 2.89 & 4.25 & 2.87 & 3.12 & 3.80 \\
\hline $1980-81$ & 3.67 & 4.63 & 4.21 & 5.45 & 3.92 & 3.46 & 4.22 \\
\hline $1981-82$ & 4.27 & 4.29 & 4.01 & 6.23 & 3.58 & 1.92 & 2.80 \\
\hline $1982-83$ & 4.88 & 5.41 & 3.99 & 7.57 & 4.37 & 1.51 & 3.20 \\
\hline $1983-84$ & 5.09 & 6.72 & 5.34 & 5.93 & 4.28 & 2.08 & 4.05 \\
\hline $1984-85$ & 7.30 & 6.88 & 5.59 & 15.91 & 7.08 & 3.02 & 4.84 \\
\hline $1985-86$ & 3.92 & 3.97 & 3.01 & 12.69 & 4.06 & 3.56 & 4.98 \\
\hline $1986-87$ & 4.56 & 6.02 & 3.60 & 10.92 & 3.72 & 4.45 & 5.80 \\
\hline $1987-88$ & 6.72 & 8.73 & 5.69 & 12.99 & 5.58 & 5.35 & 5.93 \\
\hline $1988-89$ & 6.63 & 8.41 & 5.46 & 12.64 & 6.31 & 4.33 & 4.71 \\
\hline $1989-90$ & 6.01 & 6.53 & 5.64 & 15.28 & 5.10 & 5.21 & 6.30 \\
\hline $1990-91$ & 5.38 & 6.58 & 6.31 & 17.10 & 6.11 & 4.59 & 6.85 \\
\hline $1991-92$ & 5.44 & 6.65 & 6.51 & 18.00 & 7.16 & 6.46 & 6.87 \\
\hline $1992-93$ & 3.23 & 3.88 & 2.99 & 13.75 & 3.31 & 2.22 & 3.11 \\
\hline $1993-94$ & 3.76 & 4.61 & 2.73 & 9.83 & 2.38 & 3.23 & 3.38 \\
\hline $1994-95$ & 3.25 & 4.41 & 3.47 & 11.98 & 2.64 & 2.58 & 1.66 \\
\hline $1995-96$ & 3.62 & 5.57 & 4.44 & 12.59 & 3.63 & 2.14 & 1.77 \\
\hline $1996-97$ & 3.18 & 4.07 & 3.22 & 7.99 & 2.19 & 1.12 & 1.91 \\
\hline 1997-98 & 2.81 & 4.88 & 3.07 & 8.49 & 1.66 & 0.93 & 1.50 \\
\hline 1998-99 & 4.35 & 5.58 & 5.12 & 12.07 & 4.53 & 1.95 & 2.65 \\
\hline 1999-00 & 3.19 & 4.33 & 2.55 & 6.67 & 2.52 & 3.87 & 3.36 \\
\hline $2000-01$ & 2.60 & 4.02 & 2.05 & 6.40 & 1.27 & 2.07 & 2.28 \\
\hline $2001-02$ & 2.88 & 4.20 & 2.19 & 7.81 & 2.47 & 1.96 & 2.54 \\
\hline $2002-03$ & 2.62 & 3.85 & 2.01 & 8.40 & 2.60 & 1.59 & 2.79 \\
\hline $2003-04$ & 2.20 & 3.64 & 1.07 & 7.46 & 7.48 & 1.88 & 3.28 \\
\hline $2004-05^{f}$ & 2.56 & 4.34 & 2.48 & 12.02 & 2.45 & 11.95 & 13.65 \\
\hline
\end{tabular}

${ }^{\mathrm{a}}$ On-tree average price per box (1-3/5 bushel box equivalent) for all methods of sale minus pick and haul charges.

${ }^{b}$ Navel and Hamlin $\quad{ }^{c}$ Parson Brown and Pineapple $\quad{ }^{\mathrm{d}}$ Valencia $\quad{ }^{\mathrm{e}}$ Marsh (white) or pink $\quad{ }^{\mathrm{f}}$ Preliminary

Source: Florida Agricultural Statistics Service. 
Table 16-A.--Debt which can be supported per $\$ 1,000.00$ annual payment capacity

\begin{tabular}{|c|c|c|c|c|c|c|c|c|c|c|c|c|c|c|c|}
\hline \multirow{2}{*}{$\begin{array}{c}\text { Loan } \\
\text { term } \\
\text { (years) }\end{array}$} & \multicolumn{15}{|c|}{ Interest rate paid on the loan } \\
\hline & $8.0 \%$ & $8.5 \%$ & $9.0 \%$ & $9.5 \%$ & $10.0 \%$ & $10.5 \%$ & $11.0 \%$ & $11.5 \%$ & $12.0 \%$ & $12.5 \%$ & $13.0 \%$ & $13.5 \%$ & $14.0 \%$ & $14.5 \%$ & $15.0 \%$ \\
\hline 1 & 926 & 922 & 917 & 913 & 909 & 905 & 901 & 897 & 893 & 889 & 885 & 881 & 877 & 873 & 870 \\
\hline 2 & 1,783 & 1,771 & 1,759 & 1,747 & 1,754 & 1,724 & 1,713 & 1,701 & 1,690 & 1,679 & 1,668 & 1,657 & 1,647 & 1,636 & 1,626 \\
\hline 3 & 2,577 & 2,554 & 2,531 & 2,509 & 2,487 & 2,465 & 2,444 & 2,423 & 2,402 & 2,381 & 2,361 & 2,341 & 2,322 & 2,302 & 2,283 \\
\hline 4 & 3,312 & 3,276 & 3,240 & 3,204 & 3,170 & 3,136 & 3,102 & 3,070 & 3,037 & 3,006 & 2,974 & 2,944 & 2,914 & 2,884 & 2,855 \\
\hline 5 & 3,993 & 3,941 & 3,890 & 3,840 & 3,791 & 3,743 & 3,696 & 3,650 & 3,605 & 3,561 & 3,517 & 3,475 & 3,433 & 3,392 & 3,352 \\
\hline 6 & 4,623 & 4,554 & 4,486 & 4,420 & 4,355 & 4,292 & 4,230 & 4,170 & 4,111 & 4,054 & 3,998 & 3,942 & 3,889 & 3,836 & 3,784 \\
\hline 7 & 5,206 & 5,119 & 5,033 & 4,950 & 4,868 & 4,789 & 4,712 & 4,640 & 4,564 & 4,492 & 4,423 & 4,355 & 4,288 & 4,224 & 4,160 \\
\hline 8 & 5,747 & 5,639 & 5,535 & 5,433 & 5,335 & 5,239 & 5,146 & 5,056 & 4,968 & 4,882 & 4,799 & 4,718 & 4,639 & 4,562 & 4,487 \\
\hline 9 & 6,247 & 6,119 & 5,995 & 5,875 & 5,759 & 5,646 & 5,537 & 5,431 & 5,328 & 5,228 & 5,132 & 5,038 & 4,946 & 4,858 & 4,772 \\
\hline 10 & 6,710 & 6,561 & 6,418 & 6,279 & 6,145 & 6,015 & 5,889 & 5,768 & 5,650 & 5,536 & 5,426 & 5,319 & 5,216 & 5,116 & 5,019 \\
\hline 11 & 7,139 & 6,969 & 6,805 & 6,647 & 6,495 & 6,348 & 6,207 & 6,070 & 5,938 & 5,810 & 5,687 & 5,568 & 5,453 & 5,341 & 5,234 \\
\hline 12 & 7,536 & 7,345 & 7,161 & 6,984 & 6,814 & 6,650 & 6,492 & 6,341 & 6,194 & 6,054 & 5,918 & 5,787 & 5,660 & 5,538 & 5,421 \\
\hline 13 & 7,904 & 7,691 & 7,487 & 7,291 & 7,103 & 6,923 & 6,750 & 6,583 & 6,424 & 6,270 & 6,122 & 5,979 & 5,842 & 5,710 & 5,583 \\
\hline 14 & 8,244 & 8,010 & 7,786 & 7,572 & 7,367 & 7,170 & 6,982 & 6,801 & 6,628 & 6,462 & 6,302 & 6,149 & 6,002 & 5,861 & 5,724 \\
\hline$\underline{15}$ & 8,559 & 8,304 & 8,061 & 7,828 & 7,606 & 7,394 & 7,191 & $6,997^{\mathrm{a}}$ & 6,811 & 6,633 & 6,462 & 6,299 & 6,142 & 5,992 & 5,847 \\
\hline$\overline{16}$ & 8,851 & 8,576 & 8,313 & 8,062 & 7,824 & 7,596 & 7,379 & $\overline{7,172}$ & 6,974 & 6,785 & 6,604 & 6,431 & 6,265 & 6,106 & 5,954 \\
\hline 17 & 9,122 & 8,825 & 8,543 & 8,276 & 8,022 & 7,779 & 7,549 & 7,329 & 7,119 & 6,920 & 6,729 & 6,547 & 6,373 & 6,207 & 6,048 \\
\hline 18 & 9,372 & 9,056 & 8,756 & 8,471 & 8,201 & 7,945 & 7,702 & 7,470 & 7,250 & 7,040 & 6,840 & 6,649 & 6,467 & 6,294 & 6,128 \\
\hline 19 & 9,603 & 9,268 & 8,950 & 8,650 & 8,365 & 8,095 & 7,839 & 7,596 & 7,366 & 7,146 & 6,938 & 6,739 & 6,551 & 6,370 & 6,198 \\
\hline$\underline{20}$ & 9,818 & 9,463 & 9,129 & 8,812 & 8,514 & 8,231 & 7,963 & $7,710^{\mathrm{a}}$ & 7,469 & 7,241 & 7,025 & 6,819 & 6,623 & 6,437 & 6,259 \\
\hline$\overline{25}$ & 10,675 & 10,234 & 9,823 & 9,438 & 9,077 & 8,739 & 8,422 & $\overline{8,123}$ & 7,843 & 7,579 & 7,330 & 7,095 & 6,873 & 6,663 & 6,464 \\
\hline 30 & 11,258 & 10,747 & 10,274 & 9,835 & 9,427 & 9,047 & 8,868 & 8,364 & 8,055 & 7,766 & 7,496 & 7,242 & 7,003 & 6,778 & 6,566 \\
\hline 35 & 11,655 & 11,088 & 10,567 & 10,087 & 9,644 & 9,234 & 8,855 & 8,503 & 8,175 & 7,870 & 7,586 & 7,320 & 7,070 & 6,836 & 6,617 \\
\hline 40 & 11,925 & 11,315 & 10,757 & 10,247 & 9,779 & 9,348 & 8,951 & 8,587 & 8,244 & 7,928 & 7,634 & 7,361 & 7,105 & 6,866 & 6,642 \\
\hline
\end{tabular}

${ }^{a}$ Example. Assumes a $\$ 10,000$ after tax income at $11.5 \%$ interest rate and a 15 -year term mortgage, the total debt which can be supported is $\$ 69,970$ $(\$ 6,997 \times 10)$. At $11.5 \%$ interest rate and a 20 -year term mortgage, the total debt which can be supported is $\$ 77,100(\$ 7,710 \times 10)$. 